\title{
The influence of irrelevant location information on performance: A review of the Simon and spatial Stroop effects
}

\author{
CHEN-HUI LU \\ Chinese University of Hong Kong, Shatin, Hong Kong \\ and \\ ROBERT W. PROCTOR \\ Purdue University, West Lafayette, Indiana
}

\begin{abstract}
The purpose of this paper is to investigate the effect of irrelevant location information on performance of visual choice-reaction tasks. We review empirical findings and theoretical explanations from two domains, those of the Simon effect and the spatial Stroop effect, in which stimulus location has been shown to affect reaction time when irrelevant to the task. We then integrate the findings and explanations from the two domains to clarify how and why stimulus location influences performance even when it is uninformative to the correct response. Factors that influence the processing of irrelevant location information include response modality, relative timing with respect to the relevant information, spatial coding, and allocation of attention. The most promising accounts are offered by models in which response selection is a function of (1) strength of association of the irrelevant stimulus information with the response and (2) temporal overlap of the resulting response activation with that produced by the relevant stimulus information.
\end{abstract}

The role played by stimulus location in visual information processing has been a controversial issue. This issue has been investigated primarily by means of visual search tasks in which a target stimulus in an array of distractors must be detected or identified. Reaction time (RT) in such tasks is typically an increasing function of array size, and accuracy a decreasing function, when the target is defined by a conjunction of features (e.g., Treisman \& Gelade, 1980; Van Zandt \& Townsend, 1993), leading some authors to propose that attending to location is necessary for feature integration (e.g., Nissen, 1985; Treisman \& Gelade, 1980). As expected if location must be attended in such situations, a stimulus for a second task shows a processing benefit when it occurs in a location adjacent to the target stimulus of the search task rather than in a more remote location (e.g., Hoffman \& Nelson, 1981), and precuing the location in the array in which the target stimulus will occur facilitates its processing (e.g., Eriksen \& Hoffman, 1972). However, facilitation from a location precue is also apparent when the target is presented alone and requires only a detection response (e.g., Bashinski \& Bacharach, 1980; Posner, Snyder, \& Davidson, 1980), suggesting that it may be necessary to attend to the loca-

We would like to thank Bernhard Hommel, Colin MacLeod, Bob Melara, Jim Neely, Richard Schweickert, Richard Simon, and Howard Zelaznik for helpful comments on previous versions of this manuscript. Reprint requests should be sent to Chen-Hui Lu, Department of Psychology, The Chinese University of Hong Kong, Shatin, N.T., Hong Kong, or Robert W. Proctor, Department of Psychological Sciences, Purdue University, West Lafayette, IN 47907-1364. tion of any stimulus on which a response is based. Consistent with this possibility, Tsal and Lavie (1993) recently showed that when the relevant feature of a precue stimulus is nonspatial (e.g., color), there is still facilitation in the identification of a subsequent target stimulus adjacent to the location in which the precue occurred.

That stimulus location is coded and cannot be ignored in many situations for which it is irrelevant to the task, as in Tsal and Lavie's (1993) study, has been widely documented in many choice-RT tasks. Effects of irrelevant location information on choice RTs have been demonstrated in two domains of studies, those of the Simon effect (see Simon, 1990) and the spatial Stroop effect (MacLeod, 1991). In a Simon task, the relevant stimulus dimension is a nonspatial physical feature, such as color or shape, assigned to left and right manual responses (usually keypresses), and the location (left or right) in which the stimulus occurs is irrelevant (see Figure 1, top panel). The Simon effect refers to the fact that responses are faster when the stimulus location corresponds to the location of the assigned response than when it does not. In a like version of the spatial Stroop task, stimulus location is also irrelevant, but the relevant stimulus dimension is a word or symbolic feature that conveys spatial information (see Figure 1, bottom panel). A keypress response to a location word (e.g., a left keypress to the word LEFT) is faster when the word appears at a congruent position (e.g., left side) than when it appears at an incongruent position (e.g., right side). Apparently, for both the Simon and spatial Stroop tasks, the irrelevant location information cannot be excluded from processing, even though subjects 
The Simon Effect

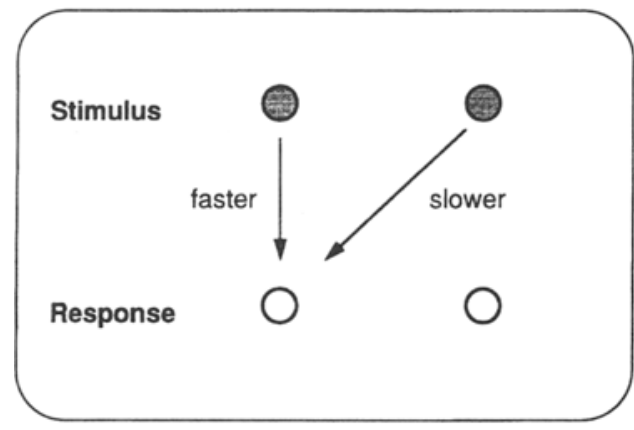

Relevant stimulus dimension: Color

Irrelevant stimulus dimension: Position

\section{The Spatial Stroop Effect}

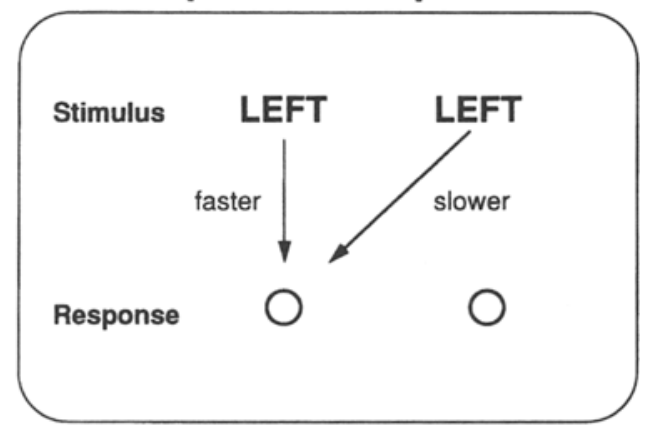

Relevant stimulus dimension: Word

Irrelevant stimulus dimension: Position

Figure 1. Examples of the Simon effect (top panel) and the spatial Stroop effect (bottom panel). For both tasks, one stimulus occurs on each trial in either a left or a right location, but stimulus location is irrelevant to the task. Keypress responses are to be based on the relevant stimulus dimension, which is unrelated to the irrelevant location dimension in the Simon task (e.g., stimulus color, as in the example) but also has a location property in the spatial Stroop task (e.g., location word, as in the example). If the left keypress response is to be made to a specific stimulus color (the Simon task) or to the word LEFT (the spatial Stroop task), responses are faster when the stimulus occurs in the left location than when it occurs in the right location (as indicated by the labeled arrows).

are instructed to respond on the basis of the relevant stimulus dimension.

The question of why location information affects responding when it is irrelevant to the task has provoked much interest, as witnessed, for example, by a recent special issue of the journal Psychological Research/Psychologische Forschung (April 1994) devoted to the Simon effect. This question provokes interest because the answer to it likely will tell us much about how stimulus properties enter into the selection of action. Although considerable research has been conducted within the domains of the Simon and spatial Stroop effects, as Umiltà (1994) noted in the introduction to the special issue, "the Simon effect is very seldom included among the Stroop-like effects" (p. 127), and little attempt has been made to relate and integrate the findings across the domains. One reason for this lack of systematic integration is that the two domains have their origins in different research literatures, those of stimulus-response (S-R) compatibility effects (Proctor \& Reeve, 1990) and color Stroop effects (MacLeod, 1991), respectively. Moreover, the Simon and spatial Stroop tasks typically have been regarded as different in nature, because the relevant and irrelevant stimulus dimensions are dissimilar in the Simon task but highly similar in the spatial Stroop task. This task distinction has been formalized in Kornblum's (1992, 1994) taxonomy, which places the Simon and Stroop tasks in different categories (Types 3 and 8, respectively) according to the similarity of the relevant and irrelevant stimulus dimensions. However, because both the Simon and spatial Stroop effects are typically attributed to responseselection processes (e.g., Dyer, 1973; Umiltà \& Nicoletti, 1990), the possibility exists that the degree of similarity between the relevant and irrelevant stimulus dimensions is not of importance.

The purpose of the present paper is to consider in detail the findings obtained for the Simon and spatial Stroop tasks, with the intent of providing a more complete understanding of the effect of irrelevant location. We first review each domain, discussing the empirical findings that have been obtained and the theoretical accounts that have been proposed for these findings. Because most recent studies and models regarding the influence of location have chiefly been concerned with visual stimuli (see, e.g., Umiltà \& Nicoletti, 1992), our review focuses primarily, but not exclusively, on visual choice-reaction tasks. For each domain, issues regarding how irrelevant location information is processed, the processing stage involved, spatial attention, and automaticity are considered. We next illustrate the similarity of the task structures for the Simon and spatial Stroop domains, as well as of the results that are obtained, and then consider several basic factors involved in the processing of irrelevant location information. The article concludes with discussion of the implications that these factors have for modeling the influence of irrelevant location information on performance.

\section{THE SIMON EFFECT}

\section{The Basic Phenomenon}

Simon and Small (1969) first obtained what has come to be called the Simon effect with auditory stimuli. ${ }^{1}$ They instructed subjects to make left or right keypresses to low- or high-pitched tones. On any trial, the tone was presented to either the left or the right ear. Responses to the "right" command (e.g., high-pitched tone) were $62 \mathrm{msec}$ faster when it was heard in the right ear rather than the left ear, whereas responses to the "left" command (e.g., low-pitched tone) were $60 \mathrm{msec}$ faster when it was heard in the left ear rather than the right ear. Simon and Small attributed this phenomenon to a natural tendency to react toward the source of stimulation. Simon and his colleagues subsequently replicated and extended this effect 
with auditory stimuli in a series of studies (see Simon, 1990 , for a review).

The effect of irrelevant location obtained with auditory stimuli is not just a transient phenomenon. Simon, Craft, and Webster (1973) conducted an experiment in which subjects responded to low- and high-pitched tones presented to the left or right ear for 216 trials on each of 5 consecutive days. RTs decreased overall with practice, but the Simon effect was not eliminated. In the first session, responses were approximately $60 \mathrm{msec}$ faster when the stimulus and response locations corresponded than when they did not. Although the magnitude of this advantage for spatially corresponding trials decreased across sessions, it was still approximately $35 \mathrm{msec}$ in the last session. Hommel (described in Prinz, Aschersleben, Hommel, \& Vogt, 1993), using a similar method, tested a single subject for 30 sessions of 210 trials each and found the Simon effect to be present in all sessions. That the Simon effect persists across relatively extensive practice suggests that it reflects a fundamental property of human information processing.

Craft and Simon (1970) provided the first demonstration of the Simon effect with visual stimuli by modifying a stereoscope to divide the visual field into left and right halves. In their Experiment 1, the stimulus was presented either monocularly or binocularly. In the monocular block, a red or green light was presented in the left visual field to the left eye or in the right visual field to the right eye. In the binocular block, two red lights or two green lights, one in each visual field, were presented simultaneously. Subjects were instructed to respond with a left-hand key to a green light and a right-hand key to a red light, or vice versa. Responses were $41 \mathrm{msec}$ faster when the right command (e.g., red light) was presented to the right eye rather than the left eye and $54 \mathrm{msec}$ faster when the left command (e.g., green light) was presented to the left eye rather than the right eye. The RTs for the binocular trials were even faster than those for the monocular trials on which stimulus and response locations corresponded, suggesting that the Simon effect is due primarily to interference that occurs when the stimulus and response locations do not correspond rather than to facilitation for trials on which they do. Whether there is a facilitatory component to the Simon effect is an issue that is not completely settled (see, e.g., Hommel, 1993b; Kornblum, Hasbroucq, \& Osman, 1990), but the term interference is often used to refer to the difference in RTs between mappings for which stimulus-response (S-R) locations do and do not correspond (e.g., Simon, Acosta, Mewaldt, \& Speidel, 1976).

The Simon effect for visual stimuli has since been replicated many times with color as the relevant stimulus dimension (e.g., Hedge \& Marsh, 1975; Simon \& Craft, 1972; Simon, Small, Ziglar, \& Craft, 1970; Umiltà \& Nicoletti, 1985). It also has been obtained with a variety of other relevant stimulus dimensions, including geometric forms (Nicoletti \& Umiltà, 1989; Umiltà \& Liotti, 1987 ) and letters (Proctor \& Lu, 1994), with wheel rotation responses (Guiard, 1983), and with vertically aligned arrangements of the stimuli and responses (Hedge \& Marsh, 1975). Although the general effect has been widely replicated, the magnitude is more typically between 20 and $30 \mathrm{msec}$, rather than the close to $50-\mathrm{msec}$ magnitude initially observed by Craft and Simon (1970).

\section{Processing Stage at Which the Effect Occurs}

Historically, the Simon effect has been considered to be a response-selection phenomenon, in part because effects of similar nature are obtained when stimulus location is the relevant attribute for determining the response. In two-choice tasks for which location is the relevant stimulus dimension, responses are faster if the left stimulus is assigned to the left response and the right stimulus to the right response than if the assignments are reversed (see, e.g., Proctor \& Dutta, 1993). Considerable evidence indicates that such spatial compatibility effects (sometimes called $S-R$ compatibility proper) are attributable to response-selection processes (see, e.g., Proctor \& Reeve, 1990). Among other things, the effects of S-R compatibility proper typically are independent from those of variables whose effects are on stimulus-identification or response-execution processes (e.g., Hasbroucq, Guiard, \& Kornblum, 1989; Spijkers, 1990). The Simon effect has similarly been found to be independent from effects of identification and execution variables, in at least some cases.

For example, Simon and Berbaum (1990) conducted experiments in which subjects pressed a left or right key in response to color Stroop stimuli (color words printed in congruent or incongruent ink colors) presented at the left or right side of the display. Half of the subjects responded to the ink color and half to the color word. Withinsubject independent variables included (1) the congruity between the relevant and irrelevant stimulus dimensions (i.e., the color word and the ink color), (2) the spatial correspondence between the irrelevant stimulus location and the response location, and (3) the stimulus duration. The results for the two task variations were similar, showing significant main effects of dimensional congruity, spatial correspondence, and stimulus duration, but no interactions. Because spatial correspondence and congruity did not interact with stimulus duration, which is customarily assumed to affect the time for stimulus identification, Simon and Berbaum concluded that neither the Simon effect nor the congruity effect were a function of stimulus-identification processes. As another example, Guiard (1983) demonstrated a Simon effect of $53 \mathrm{msec}$ for clockwise and counterclockwise wheelrotation responses made to low- and high-pitched tones presented to the left or right ear. Clockwise responses were initiated faster to stimuli presented to the right ear, whereas counterclockwise responses were initiated faster to stimuli presented to the left ear. No similar effect of the ear in which stimuli were presented was apparent in the rotation amplitudes of the responses. Guiard interpreted this pattern of results as indicating that the Simon effect is due to response selection rather than response execution. 
Most response-selection accounts of the Simon effect attribute it to response competition (Umiltà \& Nicoletti, 1990). The accounts assume that a response code is generated for the irrelevant stimulus location attribute, although they differ in the reasons proposed for how and why the code is generated. For trials on which the irrelevant response code corresponds with the response code signaled by the relevant stimulus dimension, there is no competition and possibly even a benefit from the redundant response codes. However, for trials on which the irrelevant response code does not correspond with the relevant response code, it produces competition that must be resolved before the correct response can be made. It is this response competition that is assumed to be the primary cause of the slower RTs for the noncorresponding trials relative to the corresponding trials.

Hedge and Marsh (1975) introduced a variation of the Simon task that enabled them to show that the Simon effect is not simply a function of spatial correspondence between stimulus and response locations. In their experiment, the stimulus could be either red or green, and one response key was colored red and the other green. Designating the response keys by color allowed the S-R mapping to be manipulated (see Figure 2). For the same-color mapping, subjects were to respond with the key of the same color as the stimulus, whereas for the alternatecolor mapping, they were to respond with the key opposite in color from that of the stimulus. Hedge and Marsh found a typical Simon effect of $23 \mathrm{msec}$ with the samecolor mapping but a reversed Simon effect (i.e., faster responses when the stimulus occurred in the location opposite the required response rather than in the corresponding location) of $55 \mathrm{msec}$ with the alternate-color mapping. For example, even though the correct response to a red stimulus with the alternate-color mapping is the green key, RTs were faster when the red stimulus occurred on the side of the red key than on the side of the green key.

To explain the faster responding on spatially noncorresponding trials than on corresponding trials with the alternate-color mapping, Hedge and Marsh (1975) developed a response-selection account based on the concept of logical recoding. Specifically, they proposed that the same logical recoding rule is applied to both the relevant and the irrelevant stimulus dimension. When the same-color mapping is in effect, the recoding rule is one of identity: Respond by pressing the key whose color is the same as that of the stimulus. According to Hedge and Marsh, this identity rule is also applied to the irrelevant location dimension, creating a response code for the spatially corresponding location. However, when the alternate-color mapping is in effect, the recoding rule is one of reversal: Respond by pressing the key whose color is opposite that of the stimulus. Applying this reversal transformation to the irrelevant location dimension creates a response code for the spatially noncorresponding location, thus reversing the typical Simon effect.

Simon, Sly, and Vilapakkam (1981) proposed an alternative response-selection account that attributes Hedge
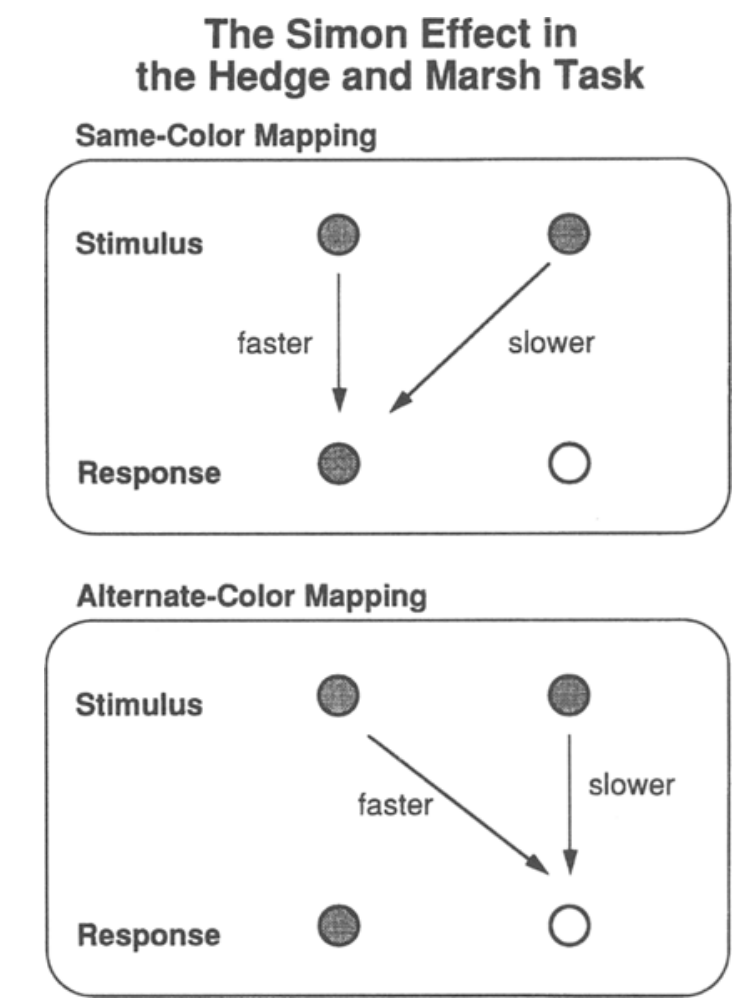

Figure 2. Schematic diagram of the Simon effect in the Hedge and Marsh (1975) task, in which the response keys are colored. For the same-color mapping, the key of the same color as the stimulus is to be pressed. For the alternate-color mapping, the key of the alternative color to the stimulus is to be pressed. In the former case, a standard Simon effect occurs, with RTs being faster when the stimulus location corresponds with that of the response than when it does not. However, in the latter case, a reversed Simon effect occurs, with RTs being faster when stimulus and response locations do not correspond than when they do.

and Marsh's (1975) results to a variation of spatial compatibility that they called display-control arrangement correspondence. This term refers to the correspondence or noncorrespondence of the spatial location of the red or green target stimulus with the location of the response key of the same color. Specifically, Simon et al. pointed out that, under the alternate-color mapping, when the stimulus is on the same side as the response, there is displaycontrol arrangement noncorrespondence (e.g., the red stimulus is on the side of the green response key), whereas when the stimulus is on the opposite side, there is display-control arrangement correspondence (e.g., the red stimulus is on the side of the red response key). Thus, they proposed that the results for the alternate-color mapping, as well as those for the same-color mapping, can be explained parsimoniously by assuming that response selection is faster when the display-control arrangement is corresponding rather than noncorresponding. Although this alternative response-selection account has not been as widely accepted as Hedge and Marsh's logical recoding account, it is still favored by Simon and his colleagues (e.g., O'Leary, Barber, \& Simon, 1994). 
The view that the Simon effect is a response-selection phenomenon has been challenged recently by Hasbroucq and Guiard (1991; see also Stoffels, Van der Molen, \& Keuss, 1989), who have proposed instead that the effect is due to stimulus-identification processes. They asserted that the Simon effect is a function of stimulus-stimulus (S-S) congruity, the correspondence between the two dimensions of the stimulus. According to Hasbroucq and Guiard, the assignment of a stimulus property (e.g., the color red) to a response position (e.g., right) by task instructions defines the property as signifying that position. Then, "because the stimulus event amounts to the presentation of two simultaneous left-right messages, it must be either intrinsically congruent or intrinsically incongruent" (Hasbroucq \& Guiard, 1991, p. 250). Stimulus identification is prolonged when the irrelevant location (e.g., left position) of a stimulus is incongruent with the relevant location (e.g., right position) signified by the relevant dimension (e.g., red color).

Hasbroucq and Guiard (1991) pointed out that the standard Simon task confounds the factor of spatial correspondence between the irrelevant stimulus location and the response location with that of the intrinsic congruity between the relevant and irrelevant stimulus dimensions. They argued that this confounding is also present in the same-color mapping condition of the Hedge and Marsh task but is eliminated in the alternate-color mapping condition. According to Hasbroucq and Guiard, because each colored response key in the Hedge and Marsh task is at a unique location, selection of a color response amounts to selection of its response location. Thus, for example, if the red response key is at the right location, the color red comes to signify "right" in a manner analogous to the way described above for the Simon task. For both the same-color and the alternate-color mappings, a red stimulus at the right location thus would be S-S congruent. With the same-color mapping, the response to this stimulus would be the red key on the right side; consequently, the stimulus location would also correspond with that of the response, and S-S congruity would be confounded with spatial correspondence. With the alternate-color mapping, however, the correct response would be the green key on the left side; the stimulus location would not correspond with that of the response, and S-S congruity would be dissociated from spatial correspondence.

Hasbroucq and Guiard's (1991) stimulus-identification account predicts that the reversed Simon effect for the alternate-color mapping in the Hedge and Marsh task should be the same magnitude as the Simon effect for the same-color mapping, because the duration of stimulusidentification processes should be unaffected by $S-R$ mapping. Their Experiment 1 was a direct methodological replication of Hedge and Marsh's (1975) experiment, except that a hand-held apparatus was used so that the colors labeling the response keys would be in the field of view when the target stimulus was presented (see Figure 3). We describe this apparatus here, because evidence discussed later in this section suggests that it may be cru- cial to the results obtained in Hasbroucq and Guiard's second experiment. The apparatus was a small box containing a row of three yellow light-emitting diodes (LEDs) that lit up as a warning, two bicolor LEDs (red or green) immediately below the left and right lights of the warning row that remained permanently lit as the color labels for the response keys (which were operated by the thumbs), and two bicolor LEDs immediately above the left and right lights of the warning row, one of which was lit as either red or green for the target stimulus. As predicted, Hasbroucq and Guiard showed a 35-msec Simon effect for the same-color mapping and a reversed Simon effect of similar magnitude $(29 \mathrm{msec})$ for the alternatecolor mapping.

According to Hasbroucq and Guiard's (1991) stimulus-identification account, when there is no correlation between the color of the response key and its location, color should not signify location and, hence, no Simon effect should occur. This relatively strong and unique prediction was tested in their Experiment 2, in which S-R correspondence was manipulated in the absence of S-S congruity by randomly varying from trial to trial whether the left key was red and the right key green, or vice versa. This was accomplished by having the LEDs designating the key colors to onset simultaneously with the target stimulus. In this case, for which there was no consistent association between color and response location, no Simon effect was apparent. Because the findings of their two experiments were in agreement with their S-S congruity account, Hasbroucq and Guiard concluded that stimulus identification is the likely locus of the Simon effect.

However, the view that the Simon effect is a function of stimulus-identification processes has not gained wide acceptance for both logical and empirical reasons. Logically, there is no inherent congruity or incongruity between the nonspatial relevant stimulus dimension and the spatial irrelevant stimulus dimension. Consequently, Hasbroucq and Guiard (1991) had to assume that stimulus identification for the relevant dimension in the stan-

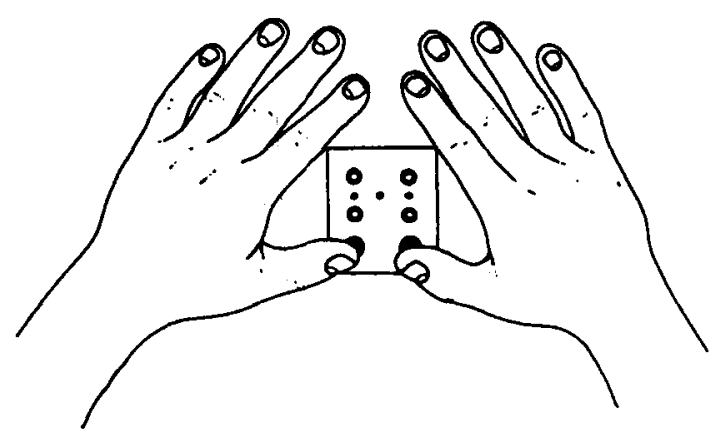

Figure 3. The hand-held apparatus used by Hasbroucq and Guiard (1991). See the text for details. From "Stimulus-Response Compatibility and the Simon Effect: Toward a Conceptual Clarification," by T. Hasbmucq \& Y. Guiard, 1991, Journal of Experimental Psychology: Human Perception \& Performance, 17, 246-266. Copyright 1991 by the American Psychological Association. Reprinted by permission. 
dard Simon task is in terms of the response location to which the stimulus is assigned by the task instructions, rather than in terms of the dimension itself (e.g., a red stimulus assigned to the right response is not identified as "red" but as "right"). The situation becomes even more problematic when the stimulus-identification account is applied to the Hedge and Marsh task, as emphasized by O'Leary et al. (1994) and Barber, O'Leary, and Simon (1994). In this task, the instructions are to respond with either the key of the same color as the stimulus or the key of the alternate color, so the instructions do not directly link stimulus color with response location. In terms of instructions, then, there is no reason for stimulus color to signify location and, hence, no basis for conflict between the relevant color and irrelevant location dimensions. Consequently, Hasbroucq and Guiard had to rely on the relation between the colors of the response keys and their physical locations to justify why color comes to signify location. Guiard, Hasbroucq, and Possamai (1994) indicated that they do not view these and other problems noted by Barber et al. as serious, but we think that they are.

Empirically, in the Hedge and Marsh task, the reversed Simon effect for the alternate-color mapping is not always the same magnitude as the Simon effect for the same-color mapping, as predicted by Hasbroucq and Guiard's (1991) stimulus-identification account and as found in their Experiment 1. For example, in Hedge and Marsh's (1975) original study described earlier, the reversed effect was over twice as large as the regular effect. $\mathrm{Lu}$ and Proctor (1994) established, across experiments in which various combinations of color and color-word stimuli and response keys labeled with colors and color words were used, that when stimulus identification was relatively slow (and, hence, RT prolonged), the magnitude of the Simon effect decreased, but that of the reversed Simon effect increased. De Jong, Liang, and Lauber (1994) demonstrated that similar opposing effects are evident within a single experimental condition when RT distributions are examined. They partitioned the RT distributions for the same-color and alternatecolor mappings into five $20 \%$ bins from fastest to slowest. The magnitude of the Simon effect for the samecolor mapping decreased from approximately $30 \mathrm{msec}$ in the fastest bin to approximately $0 \mathrm{msec}$ in the slowest bin, whereas the magnitude of the reversed Simon effect for the alternate-color mapping increased from approximately $25 \mathrm{msec}$ to approximately $55 \mathrm{msec}$. Moreover, these opposing patterns were obtained for situations in which the color of the response keys varied randomly from trial to trial, as in Hasbroucq and Guiard's Experiment 2 , which according to them should not produce either a Simon effect or a reversed Simon effect.

A major difference in the method used by De Jong et al. (1994) from that used by Hasbroucq and Guiard (1991) in their Experiment 2, which showed no significant Simon effect, was that the colors labeling the response keys appeared 1,500 msec before the target stimulus, rather than simultaneously with it. But Hommel (in press) demonstrated that even when the colors of the two response keys are not indicated until the target stimulus appears, the Simon effect can occur. He obtained significant Simon effects in three of four such experiments, with the one exception being when he used an apparatus that was virtually identical to that used by Hasbroucq and Guiard (see Figure 3). In postexperimental interviews, the subjects in that experiment indicated that they perceived the stimulus light and the response key light of the same color to form a line. On corresponding trials, the perceived line was one side or the other of the display, whereas on noncorresponding trials, it was one of the two diagonals. The important point is that for both corresponding and noncorresponding trials, the perceived line pointed to the correct response and thus provided a relatively direct cue for responding in both cases. Hommel conducted another experiment with a modified version of the apparatus intended to eliminate the diagonal line cues on the noncorresponding trials by inducing a different perceptual organization. Black foil was taped over much of the apparatus such that the middle light of the three fixation lights was covered and the two sides of the display were grouped into distinct columns. With this change in the apparatus, a Simon effect of $20 \mathrm{msec}$ was obtained. In summary, contrary to Hasbroucq and Guiard's stimulus-identification account, the Simon effect occurs in many situations for which there is no consistent association between the color assigned to a response key and the key's location, and their finding of no effect appears to be due to an unintended configural cue produced by their apparatus.

Additional data problematic for Hasbroucq and Guiard's (1991) stimulus-identification account come from tests conducted by Hommel (in press) with a procedure in which a central target stimulus that identified the response was presented approximately $1 \mathrm{sec}$ before a green "go" or red "no-go" signal that indicated whether the response was to be made. The location of the go/no-go signal varied randomly between left and right positions. In this situation, identification of the information for determining the response should have been completed before the go or no-go signal, and these signals were not correlated with response locations. Hence, according to the stimulus-identification account, there should have been no Simon effect. Yet, an effect of $42 \mathrm{msec}$ was obtained.

Given the logical and empirical problems associated with the stimulus-identification account, it is fair to conclude, as Hommel (in press) did, that "the perceptual approach to the Simon effect is not a convincing alternative to response-related theories." At present, there is relatively little reason to doubt that the Simon effect has its basis primarily in response-selection processes.

\section{Response-Selection Accounts}

Three general types of response-selection accounts, emphasizing attentional orienting, spatial coding, and overlap between the irrelevant stimulus dimension and the response dimension, have been proposed for the Simon effect (Umiltà \& Nicoletti, 1990). Simon and his 
colleagues (e.g., Simon \& Small, 1969) initially proposed an attentional orienting account in which the onset of the stimulus tends to evoke a response in the direction of the stimulus location. Mewaldt, Connelly, and Simon (1980) elaborated this account in the form of a search model in which a response buffer is formed for each possible response. These buffers, which contain all of the stimuli assigned to the respective responses, are searched in a serial, self-terminating manner. The onset of the stimulus is assumed to orient the subject to search first the buffer that corresponds to the stimulus location. Verfaellie, Bowers, and Heilman (1988) modified the attentional orienting account by attributing the advantage for responses on the same side as the stimulus to patterns of hemispheric activation. Specifically, they proposed that when a stimulus is presented in one hemispace, the contralateral cerebral hemisphere is activated and attention is directed toward the corresponding hemispace. The response in that hemispace will have an advantage because it is mediated by the activated hemisphere. These attentional orienting accounts are not given much credence currently because ad hoc assumptions are required to explain the fact that the Simon effect can be obtained when the relative left/right stimulus and/or response locations are both in the same hemispace (e.g., Umiltà \& Nicoletti, 1985 ; this point is discussed in more detail in the subsequent section, Spatial Coding). However, as discussed later, a currently popular view is that attention shifts play a role in spatial coding.

Spatial coding accounts have been advocated for both spatial S-R compatibility effects proper (e.g., Nicoletti, Anzola, Luppino, Rizzolatti, \& Umiltà, 1982) and the Simon effect (e.g., Umiltà \& Nicoletti, 1985; Wallace, 1971). When stimulus location is relevant, as is the case for S-R compatibility effects proper, the view is that a spatial stimulus code is formed and a spatial response code is selected. When stimulus location is irrelevant, as in the Simon effect, a spatial code in response to stimulus location is presumed still to be formed, along with a spatial code in response to the relevant stimulus dimension. Response selection is slowed when the two spatial codes indicate different response locations rather than the same location.

The third type of account, that of dimensional overlap, focuses on the similarity between stimulus dimensions and response dimensions. According to Kornblum et al.'s (1990) dimensional overlap model, a stimulus automatically activates its corresponding response when there is dimensional overlap (i.e., similarity) between the stimulus and response dimensions. For the Simon task, the irrelevant dimension of stimulus location overlaps with the response location dimension. Consequently, the response corresponding to the location of the stimulus will be automatically activated. Responding will be speeded when this response is the same as the one indicated by the relevant stimulus dimension but slowed when the two conflict.

Spatial coding accounts of the Simon effect have been most widely accepted because, as described in the fol- lowing sections, the Simon effect does not depend on absolute locations of the stimuli or the responses, or on which hand is operating which response key. Most recent studies therefore have examined the nature of the spatial coding process. Our remaining discussion of the Simon effect focuses on the results of these studies.

\section{Spatial Coding}

If spatial coding is important, no Simon effect should be obtained when the stimuli are presented to either the left or the right eye but are perceived to occur at the same location. Craft and Simon (1970), in their investigation that first established the occurrence of the Simon effect for visual stimuli, examined in their Experiment 2 whether the eye stimulated per se or the perceived location of the stimulus was more crucial. A modified viewer allowed the stimulus to be presented to the subject's left or right eye but to be seen in the center of the visual field. No Simon effect was obtained for this situation in which the eye that was stimulated varied but the perceived location did not. This outcome would be expected if spatial coding of stimulus location underlies the Simon effect, because the arrangement allowed no opportunity for the formation of left-right spatial codes.

Spatial coding is important not only for the stimulus display but also for the response set. For the tasks showing the Simon effect, spatial responses (e.g., left or right response) are required. Simon, Hinrichs, and Craft (1970), using auditory stimuli, and Wallace (1971), using visual stimuli, showed that the Simon effect is independent of the limb used to make the response. Subjects in both studies were instructed to make left/right keypress responses to the stimulus with either uncrossed (left hand on left key and right hand on right key) or crossed (left hand on right key and right hand on left key) hand placements. Although the responses were slower overall when the hands were crossed, the magnitude of the Simon effect was unaffected. Wallace (1972) conducted a similar experiment in which subjects could not see the positions of their upper limbs. The Simon effect was still obtained for both crossed and uncrossed hand placements, even though the positions of the responding hands were specified by kinesthetic cues alone. Wallace (1972) concluded that the Simon effect is a function of coding the responses, as well as the stimuli, in terms of left and right spatial locations.

Although spatial coding of response locations is crucial to the Simon effect, evidence suggests that this coding is not always with respect to the response key that is operated. Hommel (1993b) used an auditory version of the Simon task in which a high or low pitch tone, to be identified by pressing a left or right key, was presented in a left or right location. The unique feature of his procedure was that the keypress activated a left or right light. Of most concern are his conditions in which the light-key mapping was inverted such that the left key lit the right light and the right key lit the left light. Subjects instructed to respond to each tone stimulus by pressing the assigned response key showed a Simon effect of 
52 msec. However, subjects instructed to respond to the tone stimulus by producing the assigned light showed a reversed Simon effect of $30 \mathrm{msec}$. In other words, when instructed in terms of the goal of activating the assigned light, responses were faster when the location of the action goal (e.g., the right light) corresponded with the location of the stimulus (e.g., the low pitch tone presented to the right), even though the location of the key that was pressed did not correspond with the stimulus location. This outcome is consistent with the finding of Riggio, Gawryszewski, and Umiltà (1986) that, for S-R compatibility proper, when subjects respond with sticks that are crossed such that movement of the hand on one side operates the key on the opposite side, compatibility is a function of the correspondence between the stimulus location and the location of the end of the stick - that is, the action goal. The fact that the Simon effect was reduced in Hommel's study when the instructions involved the light, relative to when they involved the response key, suggests that other correspondences (e.g., that between the stimulus location and the key location) may contribute to the Simon effect as well, and Hommel reported some additional support for this conjecture.

Spatial coding of the responses influences RT even when the response alternatives are not directly defined by spatial features. In the Hedge and Marsh (1975) variation of the Simon task, described earlier, colored stimuli are assigned to colored response keys on the left and right. Although the $S-R$ mappings are defined by the color relations (e.g., press the green key to the red stimulus and the red key to the green stimulus), the different colored response keys are still distinguished according to spatial location (e.g., the green key is left and the red key is right). The Simon effect is obtained for this case, in which the responses are not defined by spatial locations but still have a spatial attribute (Brebner, 1979; De Jong et al. 1994; Hasbroucq \& Guiard, 1991; Hedge \& Marsh, 1975; Lu \& Proctor, 1994; Simon et al., 1981), suggesting that the Simon effect occurs whenever the response must be coded spatially.

Umiltà and Nicoletti (1985) established that the spatial codes underlying the Simon effect can be based on relative location. For one block of trials, both stimulus locations and both response locations were to the left of body midline; for another block, they were to the right of midline. The Simon effect was obtained as a function of the relative locations of the stimuli and responses. Regardless of whether the stimulus and response locations were both in the right or both in the left hemispace, responses to the rightmost stimulus were faster when made with the right finger than when made with the left finger, and vice versa for the leftmost stimulus. The Simon effect of approximately $25 \mathrm{msec}$ when both stimulus locations were in the same hemispace was similar in magnitude to the effect of $22 \mathrm{msec}$ that Umiltà and Nicoletti obtained in a control experiment in which the stimulus locations were in different hemispaces. One implication of this result is that relative location, rather than hemispace, is more crucial to the Simon effect.
Umiltà and Liotti's (1987) Experiment 3 similarly demonstrated the contribution of relative position coding to the Simon effect, but using a precuing paradigm. The hemispace in which the target would appear was indicated by presenting two boxes to the left or right of a central fixation (see Figure 4, top). The target stimulus, a square or oblong rectangle, occurred inside one of the two boxes either simultaneous with the onset of the boxes or $500 \mathrm{msec}$ later. No Simon effect was evident when the two boxes and the target appeared simultaneously. However, when the boxes designating the hemispace appeared prior to the target, a Simon effect of $21 \mathrm{msec}$ was obtained as a function of relative position. Umiltà and Liotti (1987) obtained comparable results in another experiment, which they interpreted as indicating that spatial coding can also be based on hemispace. In their Experiment 4 , the possible target locations were designated by two solid-contour boxes, one to each side of fixation; the two remaining locations were marked by brokencontour boxes (see Figure 4, bottom). When the onset of the boxes preceded that of the target by $500 \mathrm{msec}$, a Simon effect of $21 \mathrm{msec}$ again was obtained -in this case, as a function of whether the stimulus occurred in the left or right hemispace.

Umiltà and Liotti's (1987) results suggest that the spatial code can be formed in terms of egocentric hemispace as well as relative stimulus location. Lamberts, Tavernier, and d'Ydewalle (1992) also obtained results suggesting that the Simon effect can be based on multiple spatial codes. In their Experiment 2, the locations of eight boxes were manipulated according to three factors (see Figure 5): the hemispace of stimulus presentation (left or right of center), visual hemifield within hemispace (left or right of a fixation cross located in the hemispace), and relative position within hemifield (left

\section{Experiment 3}

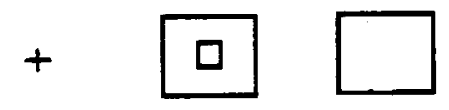

\section{Experiment 4}

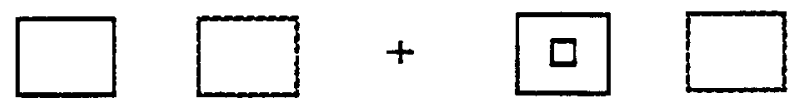

Figure 4. Schematic diagram of the stimulus displays for Experiments 3 and 4 of Umiltà and Liotti (1987). The boxes drawn with solid lines indicate the precued locations. In Experiment 3, the precue boxes were displayed to the right (as illustrated) or left of fixation. In Experiment 4, the precue boxes were displayed either in the two locations indicated by the bold lines or in the other two locations. Boxes were also displayed at the uncued locations, but with broken contours. The subject's task in both experiments was to indicate whether the target stimulus, which occurred in one of the two cued boxes, was a square or oblong rectangle. 


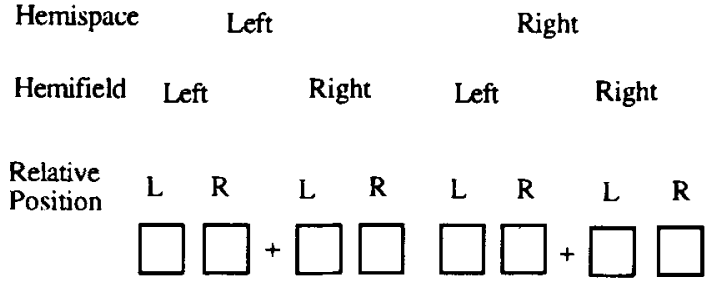

Figure 5. Schematic diagram of stimulus locations for Lamberts, Tavernier, and d'Ydewalle's (1992) study. At the beginning of each trial, a fixation cross was presented in either the left or the right half of the display for $500 \mathrm{msec}$. At the offset of the fixation, two boxes were shown, both either to the left or to the right of the fixated location, and the target stimulus (a square or circle) occurred in one of them. From "Effects of Multiple Reference Points in Spatial Stimulus-Response Compatibility," by K. Lamberts, G. Tavernier, \& G. d'Ydewalle, 1992, Acta Psychologica, 79, 115-130, Figure 1. Copyright 1992 by Elsevier Science Publishers B. V. Reprinted by permission.

or right). At the beginning of each trial, one of the two fixation crosses was presented for $500 \mathrm{msec}$ to precue the hemispace in which the stimulus would be presented. At its offset, two boxes appeared on the same side, either left or right of the fixation location, indicating the hemifield of stimulus presentation. At the same time, the target stimulus (a square or circle) was presented within one of the two boxes.

The three variables of hemispace, hemifield, and relative position had additive contributions to the Simon effect of 8,15 , and $22 \mathrm{msec}$, respectively. That is, RT was slower with the right response than with the left response to a left-hemispace stimulus, left-hemifield stimulus, and left relative-position stimulus, whereas the opposite relation held for a right-hemispace stimulus, right-hemifield stimulus, and right relative-position stimulus. In other words, the Simon effect was largest for the leftmost and rightmost stimulus locations. It is surprising that Lamberts et al. (1992) obtained Simon effects in their experiment because the display format (i.e., simultaneous presentation of two boxes and a target stimulus to either side of a fixation point) was similar to the format used by Umiltà and Liotti (1987) with centered displays that showed no Simon effect for simultaneous presentation. Hommel (1994a) proposed a possible resolution to this discrepancy in terms of relative processing speed for the relevant and irrelevant dimensions, which will be described in the section Relative Processing Speed.

\section{Attention Shifting Account}

Some studies suggest that the frame of reference for spatial coding may be provided by the location at which attention is directed. Nicoletti and Umiltà (1989) had subjects make a left or right keypress response to a rectangle or square that appeared inside one of six boxes arranged in a row (see Figure 6). In their Experiment 3, subjects were instructed to maintain fixation on a plus sign located at one end of the row and to orient attention onto a small solid square that was shown for $500 \mathrm{msec}$ in one of the five gaps between the boxes. Five hundred milliseconds after the offset of the square, the imperative stimulus occurred in one of the immediately adjacent boxes. A Simon effect averaging $48 \mathrm{msec}$ occurred with respect to the location at which attention was oriented. That is, regardless of where the orienting square was placed, a Simon effect was obtained for the two adjacent stimulus locations. Similar results were obtained in their Experiment 4, even though the stimulus array was partitioned into two parts by a large gap separating the two middle boxes. From these results, Nicoletti and Umiltà concluded that a right-left subdivision of space and the Simon effect occur with respect to the location at which attention is oriented.

Nicoletti and Umiltà (1994) provided converging evidence that attentional orienting yields the spatial code that causes the Simon effect by showing that the effect is not obtained when attentional focus must remain at fixation. The display was identical to that used by Nicoletti and Umiltà (1989) with three major exceptions: (1) no solid square was presented to direct attention, (2) the fixation cross was presented at a central location rather than at either side, and (3) a letter (X, N, H, or W) was shown below the fixation cross for $100 \mathrm{msec}$ simultaneously with the target stimulus. The letter $\mathrm{W}$ indicated a catch trial to refrain from responding to the target. Therefore, subjects needed to keep attention at fixation to detect the letter and could not voluntarily direct attention to the position of the target. This procedure, which required attention to be focused on the letter at fixation, likely prevented involuntary shifts of attention to the target as well (Yantis \& Jonides, 1990). The results showed no Simon effect for this condition, in which movement of attention to the target stimulus was prevented. In another experiment, Nicoletti and Umiltà (1994) presented the letter indicating whether or not to respond $500 \mathrm{msec}$ before the onset of the target stimulus. In this case, a Simon effect of $18 \mathrm{msec}$ was obtained with respect to the center of the display. Thus, when the letter discrimination could be performed before the reaction task, allowing attention subsequently to be shifted to the position of the target stimulus, the Simon effect occurred.

Stoffer (1991) proposed that, with the procedure used by Umiltà and Liotti (1987, Experiment 3 ) in which two boxes to the left or right of fixation designate the hemispace in which the target stimulus will occur, the Simon

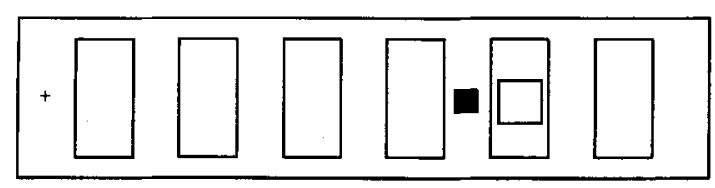

Figure 6. Schematic drawing of the displays used in Nicoletti and Umilta's (1989) Experiments 3 and 4. The six empty boxes were always present, and a fixation cross was located at the left (as illustrated) or right of the display. A small filled square came on in one of the five gaps between boxes for $500 \mathrm{msec}$ prior to a trial, and the target stimulus-either an oblong rectangle or a square-was presented in one of the two adjacent boxes. The subject was instructed to fixate the cross but to orient attention on the location indicated by the small filled square. 
effect is determined by whether attention is shifted from the central fixation to the two boxes prior to onset of the target. In Stoffer's account, attention can be directed to different hierarchical levels of visual representation, with the spatial codes that cause the Simon effect being produced when attention shifts laterally within a single representational level. Precuing the hemispace with two boxes allows the focus of attention to be shifted from the fixation point to a location between the boxes. When the target is subsequently presented, the attentional focus shifts laterally from the location between the boxes to the target, producing a spatial code corresponding to the direction of the shift. If there is no time for attentional focus to be switched from the fixation point to the location between the two boxes prior to presentation of the target, as is the case when the target is presented simultaneously with the boxes, no Simon effect would occur.

Furthermore, Stoffer (1991) proposed that the Simon effect should not occur when attention zooms in on a subordinate representational level. He conducted an experiment that replicated Umiltà and Liotti's (1987) condition in which the cued locations were designated by two boxes (Figure 7, small cue condition) and also included a condition in which the cued locations were designated by a large rectangle surrounding the two possible stimulus positions (Figure 7, large cue condition). The small cue condition yielded results similar to those obtained by Umiltà and Liotti: the Simon effect was a negligible $9 \mathrm{msec}$ when the cue occurred simultaneously with the target, but the effect was $51 \mathrm{msec}$ when the cue preceded the target by $500 \mathrm{msec}$. In contrast, in the large cue condition, the Simon effect was not statistically reliable either when the cue occurred simultaneously with the target ( $-1-\mathrm{msec}$ effect) or when it preceded the target (3-msec effect). According to Stoffer, the Simon effect is absent when the possible stimulus locations are

\section{Small cue condition}

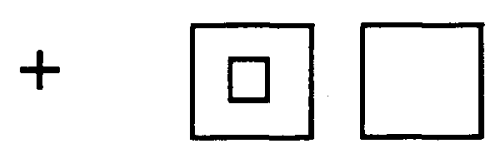

\section{Large cue condition}

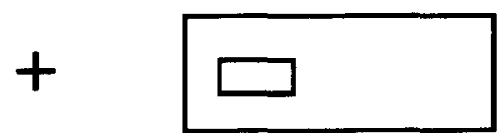

Figure 7. Examples of the stimulus displays used in Stoffer's (1991) experiment. For the small cue condition, the precue was two boxes to the left or right of a fixation cross. For the large cue condition, the precue was a single large box that covered the same area as the two boxes in the small cue condition. In both cases, the target stimulus was either a square or an oblong rectangle. precued with the large rectangle because the final step in attentional refocusing involves zooming in onto a subordinate representational level and, hence, produces no left-right spatial code.

It should be noted, however, that Weeks, Chua, and Hamblin (in press) were not able to replicate the striking interaction obtained by Stoffer (1991). In an experiment designed to be virtually identical to his, they also found the Simon effect to be larger when the cue-target delay was $500 \mathrm{msec}$ than when it was $0 \mathrm{msec}$. However, in contrast to Stoffer's results, a significant Simon effect of $18 \mathrm{msec}$ was evident for the large cue at the $500-\mathrm{msec}$ cue-target delay. This effect was only slightly less than the 29-msec effect obtained with the small cue at the same delay, and the interaction between cue type and cue-target delay predicted by the attention shifting account was not significant. In two subsequent experiments, Weeks et al. showed reliable Simon effects of similar magnitudes for the large and small cue conditions when (1) only the 500-msec delay was used and (2) the cues were centered centrally. The data of Weeks et al. thus provide little evidence of the attentional zooming mechanism hypothesized by Stoffer.

\section{Referential Coding Account}

Hommel (1993d) has argued against the attention shifting account and proposed instead that the location of the stimulus is coded in terms of relative position with respect to a reference object or frame. He conducted experiments in which the target stimulus (e.g., a red or green colored rectangle in his Experiment 1) was accompanied by an extra, noninformative stimulus (e.g., a gray rectangle) in the location not occupied by the target, to serve as a referent. A large single frame surrounding both possible stimulus locations was presented before the onset of the target stimulus and the noninformative stimulus to evaluate Stoffer's (1991) view that no spatial code is produced when attention must zoom in from a frame to the target location. The Simon effect was found in all of Hommel's experiments in which the target stimulus occurred in the context of a reference object. Hommel interpreted these results as indicating that spatial stimulus coding depends on the availability of a referent object or frame of reference, which in the standard paradigm may be provided by the fixation point.

Grice and his colleagues (Grice, Canham, \& Boroughs, 1984; Grice, Canham, \& Gwynne, 1984) obtained similar results for control conditions included to evaluate the influence of redundant targets on RTs. In their studies, two letters were presented, one to the right and one to the left of a central fixation. On redundant target trials, the same target letter (either an $\mathrm{H}$ or an $\mathrm{S}$ ) was presented in both locations. For single-target trials, an irrelevant letter $(\mathrm{Y})$ was presented in the location opposite the target. For the single-target condition, the target location either corresponded with the response or did not. A significant Simon effect was obtained, and Grice, Canham, and Boroughs found the magnitude to be greater with a wide spacing of $6^{\circ}$ of visual angle ( $45 \mathrm{msec}$ ) between stimuli 
than with a smaller spacing of $0.5^{\circ}(23 \mathrm{msec})$. However, using a similar procedure, Proctor and $\mathrm{Lu}$ (1994) found separations of $6^{\circ}$ and $2.9^{\circ}$ to yield Simon effects of 20 and $21 \mathrm{msec}$, respectively-values that not only do not differ significantly but that also are similar to that obtained by Grice, Canham, and Boroughs at the $0.5^{\circ}$ separation. Thus, the evidence regarding whether targetdistractor spacing influences the magnitude of the Simon effect obtained with Grice's procedure is ambiguous.

Proctor and Lu (1994) also obtained several results with variations of Grice's procedure that are inconsistent with the view that location coding of the target occurs with respect to a referent object. First, the Simon effect without a noise stimulus was larger when there was no fixation point than when there was (19 vs. $6 \mathrm{msec}$ in their Experiment 3 ). Because the fixation point provides a relatively obvious referent with respect to which the stimulus location could be coded as left or right, it seems that the Simon effect should have been at least as large with a fixation as without. Second, when a fixation point was present, inclusion of the noise stimulus in the location opposite that of the target increased the magnitude of the Simon effect (from 6 to $18 \mathrm{msec}$ in Experiment 2), whereas it did not do so when a fixation point was not present ( 19 vs. $21 \mathrm{msec}$ ). Because there was already a reference object (the fixation point) in the former situation but not in the latter, the referential coding account would seem to predict that the relative effects of the noise stimulus should have been the opposite. Third, presenting the target and noise letters in different colors substantially reduced the magnitude of the Simon effect ( 3 vs. $25 \mathrm{msec}$ in Experiment 4). There is no reason to expect such a result if the noise stimulus were serving as a referent for spatial coding. Instead, the noise stimulus appears to act as a distractor from which the target must be distinguished, with this stimulus-selection problem being minimized if the target and noise differ on a salient physical feature.

The results of Proctor and Lu's (1994) experiments seem difficult to reconcile with the simple notion that the Simon effect arises from location coding with respect to prominent referent objects. However, this does not mean that a more fully articulated referential coding account will necessarily be inadequate. An infinite number of reference frames may be adopted for any given display (Logan, 1994), which suggests that many factors may contribute in a complex manner to referential coding.

\section{Relative Processing Speed}

Hommel (1993c) proposed a temporal overlap hypothesis based on the view that relative processing speed influences the magnitude of the Simon effect. According to this hypothesis, the spatial code for the irrelevant stimulus dimension is formed rapidly upon stimulus onset and then decays. The magnitude of the Simon effect is assumed to be a function of the degree of overlap in the activation functions for the irrelevant and relevant codes. Results consistent with the hypothesized decay of activation of the irrelevant spatial code are apparent in a study by Simon et al. (1976), who had subjects withhold responding to a red or green stimulus presented in the right or left visual field until a go signal (a $100-\mathrm{Hz}$ tone) was heard. When the tone occurred simultaneously with the target stimulus, a Simon effect of approximately $40 \mathrm{msec}$ was obtained. This effect decreased in magnitude as the delay between the onsets of the target and the go signal increased, with the Simon effect being completely absent at the longest delay of $350 \mathrm{msec}$.

Because the irrelevant spatial code for stimulus location is presumed to be formed rapidly and automatically, its influence on responding to the relevant stimulus information should be largest when that information can be identified quickly. Any delay in identification of the relevant stimulus dimension thus should reduce or eliminate the Simon effect. Hommel (1993c) performed tests of this prediction using monochromatic forms (letters in some experiments) as stimuli and manipulating in different experiments stimulus eccentricity, signal quality (whether the background was white, overlaid with an evenly structured gray pattern, or overlaid with a complex black-and-white pattern), and signal-background contrast. In one experiment, stimuli were presented in different blocks at lateral eccentricities of $0.2^{\circ}, 3.0^{\circ}$, and $6.0^{\circ}$. RTs were an increasing function, and the Simon effect a decreasing function, of eccentricity (effects of 23 , 11 , and $-5 \mathrm{msec}$, respectively). The manipulations of signal quality and signal-background contrast produced similar outcomes. In a final experiment, Hommel gradually presented either the letter $U$ or the letter $D$ across time, so that the location of the stimulus was available at the onset of the formation period but the information needed to identify the letter was not available until $196 \mathrm{msec}$ after the presentation was begun. Overall RTs were slower with the gradual presentation than with an abrupt presentation of the stimuli. More importantly, the Simon effect of $18 \mathrm{msec}$ obtained with gradual presentation was less than the effect of $33 \mathrm{msec}$ that was obtained with abrupt onset.

An additional finding consistent with the temporal overlap hypothesis was reported in a dual-task experiment conducted by McCann and Johnston (1992). Subjects in their experiment heard a tone to which they were to respond vocally "high" or "low" with respect to its pitch. At stimulus onset asynchronies (SOAs) varying from 50 to $800 \mathrm{msec}$, the letter $M$ or $T$, to which a left or right keypress response was to be made, occurred in a left or right location. The responses for the second task showed a typical psychological refractory period effect (Smith, 1967) - that is, the RTs were slowed greatly at short SOAs relative to long SOAs. More importantly, a Simon effect of $36 \mathrm{msec}$ was evident at the longest ( 800 $\mathrm{msec}$ ) SOA but decreased monotonically to a nonsignificant $3-\mathrm{msec}$ value at the shortest $(50-\mathrm{msec})$ SOA. This outcome is what would be expected if the formation of a spatial code in response to the relevant stimulus dimension was slowed at the short SOAs, thus producing little temporal overlap with the activation of the irrelevant spatial location code. 
The decay of the irrelevant spatial code could reflect either a spontaneous automatic process or a voluntary inhibitory strategy. Hommel (1994b) evaluated these alternatives by manipulating the relative frequency of noncorresponding to corresponding trials in an experiment in which presentation of the relevant stimulus information was either immediate (i.e., simultaneous with the irrelevant location information) or delayed by gradual presentation of the stimulus elements, as described above. Logan and Zbrodoff (1979), discussed in more detail later in the paper, demonstrated that the spatial Stroop effect can be reversed by having $80 \%$ of the trials be noncorresponding and only $20 \%$ be corresponding, implying that strategies vary as a function of the relative frequency of the two trial types. Hommel compared performance in situations in which either $80 \%$ or $20 \%$ of the trials were noncorresponding. He found a smaller Simon effect with delayed presentation than with immediate presentation, as in his previous study, and a reversal of the Simon effect when $80 \%$ of the trials were noncorresponding, similar to Logan and Zbrodoff's study. However, the influence of relative frequency on the Simon effect, which is presumed to be strategic, did not modify the influence of immediate versus delayed presentation. Consequently, Hommel concluded that the reduction in the Simon effect that occurs when the relevant information is delayed most likely does not reflect a strategic process but rather spontaneous decay of the location code.

We indicated earlier that a discrepancy exists between the studies of Umiltà and Liotti (1987) and Lamberts et al. (1992). Both included conditions in which the target stimulus was displayed within one of two boxes presented simultaneously to either the left or the right of a fixation cross, but Umiltà and Liotti found no Simon effect, whereas Lamberts et al. did. Hommel (1994a) proposed a resolution of this discrepancy in terms of the temporal overlap hypothesis. He noted that, in Umiltà and Liotti's study, the targets were two rectangles of different widths, whereas, in Lamberts et al.'s study, the targets were a rectangle and a circle. Hommel suggested that stimulus identification took longer for the narrow and wide rectangle targets than for the rectangle and circle targets, because the former are more difficult to discriminate than are the latter. Thus, the temporal overlap of the activation function for the relevant code with that for the irrelevant spatial code would have been less in Umiltà and Liotti's study than in that of Lamberts et al., accounting for the absence of a Simon effect in the former case but not in the latter case.

Hommel (1994a) manipulated the processing speed of the relevant stimulus dimension by using either narrow and wide rectangle stimuli identical to those used by Umiltà and Liotti (1987) or color stimuli (green or red). As expected, responses were slower to the rectangle stimuli than to the color stimuli. More importantly, no Simon effect was obtained with the rectangle stimuli, replicating Umiltà and Liotti's finding, whereas a significant Simon effect as a function of hemispace of $15 \mathrm{msec}$ was shown with the color stimuli. Although the Simon effect was evident for the color stimuli, as predicted by the temporal overlap hypothesis, the fact that the effect occurred with respect to hemispace does not fit with the findings of either Lamberts et al. (1992) or Umiltà and Liotti. Hommel suggested that the Simon effect for relative location within the hemispace was absent in his study because the relative location code was formed quickly and then decayed. However, because stimuli can be segregated preattentively on the basis of color (e.g., Treisman \& Gelade, 1980), another possibility is that no relative location code was ever formed (see Stoffer \& Yakin, 1994, for more discussion of this point). Likewise, the possibility exists that the Simon effect may have been completely absent for the rectangle stimuli because neither a location code with respect to relative location nor a location code with respect to hemispace was formed in that task. Thus, it is not clear that the discrepant results obtained in these studies can be attributed primarily to temporal overlap.

Even though considerable data are interpretable in terms of the temporal overlap hypothesis, the findings mentioned earlier of $\mathrm{Lu}$ and Proctor (1994) and De Jong et al. (1994) with the Hedge and Marsh task, in which the response keys are colored, indicate that the hypothesis cannot account for all relative timing results. Both of those studies showed that whereas the magnitude of the Simon effect obtained with the same-color S-R mapping decreases as RTs increase, the magnitude of the reversed Simon effect obtained with the alternate-color S-R mapping increases. De Jong et al. proposed a dualprocess account of the Simon effect to explain these opposing results of relative timing on the normal and reversed Simon effects. The first process in De Jong et al.'s (1994) model is an unconditional automatic priming of the spatially corresponding response that decays rapidly. This process is the equivalent of the activation and decay function proposed by Hommel (1993b). The second process is generalization of the S-R transformation (identity or reversal) that is defined by the task instructions: The task-defined transformation is applied not only to the relevant stimulus dimension but also to the irrelevant dimension of stimulus location, as proposed by Hedge and Marsh (1975). This generalization component is presumed not to be time-locked to stimulus onset but to arise at the time the transformation rule is applied.

Figure 8 illustrates the time courses of the contributions of the two components and the predicted changes in the magnitudes of the normal and reversed Simon effects as RT increases. Note that with same-color mapping, for which the identity transformation applies, both components favor responses at the response location that corresponds to the stimulus location; hence, the magnitude of the Simon effect decreases across time as the automatic priming decreases. However, with the alternatecolor mapping, for which the reversal rule applies, the two components favor responses at opposing locations; hence, the magnitude of the reversed Simon effect increases across time as the automatic priming decreases. These predictions coincide with the actual patterns of re- 


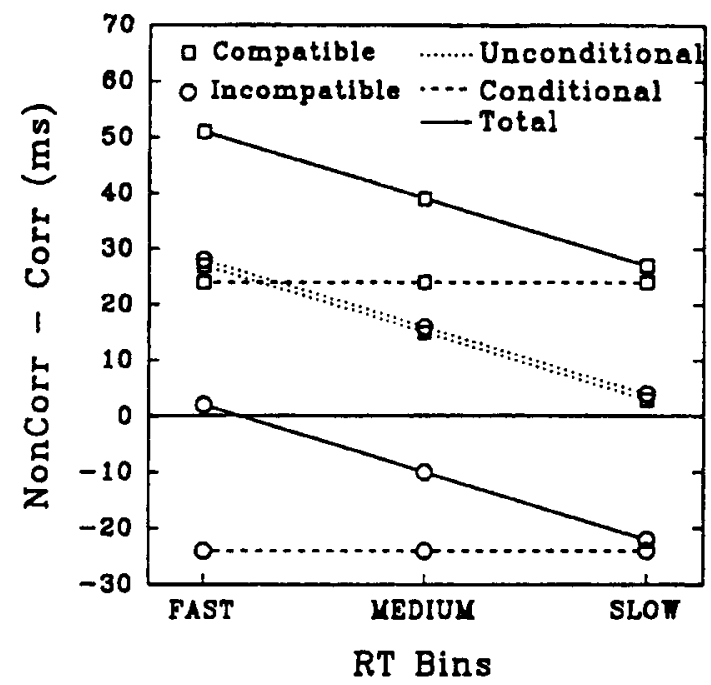

Figure 8. An illustration of the predictions of De Jong et al.s (1994) dual-process model. The same-color mapping is labeled "compatible" and designated by squares, whereas the alternate-color mapping is labeled "incompatible" and designated by circles. The unconditional component depends on relative response speed but not on the compatibility of the $\mathbf{S}-\mathbf{R}$ mapping. The conditional component depends on the mapping but not on the response speed. The overall Simon effects are obtained by adding the two components. Noncorr = noncorresponding; Corr $=$ corresponding; $\mathbf{R T}=$ reaction time. From "Conditional and Unconditional Automaticity: A Dual-Process Model of Effects of Spatial Stimulus-Response Correspondence," by R. De Jong, C.-C. Liang, \& E. Lauber, 1994, Journal of Experimental Psychology: Human Perception \& Performance, 20, 731-750, Figure 1. Copyright 1994 by the American Psychological Association. Reprinted by permission.

sults that De Jong et al. (1994) obtained, which we described in detail earlier. De Jong et al.'s model, which combines Hommel's (1993b) temporal overlap account with Hedge and Marsh's (1975) logical recoding account, thus is able to accommodate a wide range of disparate findings regarding the Simon effect.

\section{Enhancement and Reduction of the Simon Effect With Precuing}

We have already discussed the results of studies in which a subset of two or more stimulus locations from a larger set is precued. In the present section, we consider experiments in which the precue designates a single stimulus or response location as probable. Verfaellie et al. (1988) used a procedure (see Figure 9) in which they precued the likely stimulus location, the likely response location, or both. A trial began with the illumination of two central precue stimuli, one above and one below fixation. The response-location precue, located above fixation, was a hand that pointed in the direction of the likely response location or, when uninformative, a box indicating that both response locations were equally likely. The stimulus-location precue, located below fixation, was an arrow that pointed toward the likely stimulus location or, when uninformative, a box indicating that both stimulus locations were equally likely. ${ }^{2}$ Any combination of in- formative and uninformative stimulus- and responselocation precues could occur on any trial. The precues always preceded onset of the target stimulus (a bright or dim circle), which occurred to the left or right, by approximately $3 \mathrm{sec}$, and the informative precues of both types were valid on $80 \%$ of the trials and invalid on $20 \%$ of the trials.

Informative stimulus- and response-location precues both reduced RTs overall relative to when neither cue was informative, with the reduction being greater for the response-location precues than for the stimulus-location precues. However, a significant Simon effect (of $40 \mathrm{msec}$ ) was evident only for the condition in which response location alone was precued. Thus, the Simon effect was enhanced by a precue that signaled the likely response but unaffected by a precue that signaled the likely stimulus location.

An unusual feature of Verfaellie et al.'s (1988) results was the absence of the Simon effect for the condition in which neither stimulus location nor response location was precued. Because this condition provided the baseline against which the influence of the precues was evaluated, Proctor, Lu, and Van Zandt (1992) replicated Verfaellie et al.'s procedure closely using a display arrangement that differed primarily in that each type of precue was presented in one location, rather than in three different locations, and the SOA between precue and target onsets was $1 \mathrm{sec}$. Unlike Verfaellie et al., a 17-msec Simon effect was obtained in the neutral condition for which neither stimulus location nor response location was precued. However, consistent with Verfaellie et al., the magnitude of the Simon effect was enhanced only by the responselocation precue, for which the effect was $35 \mathrm{msec}$.

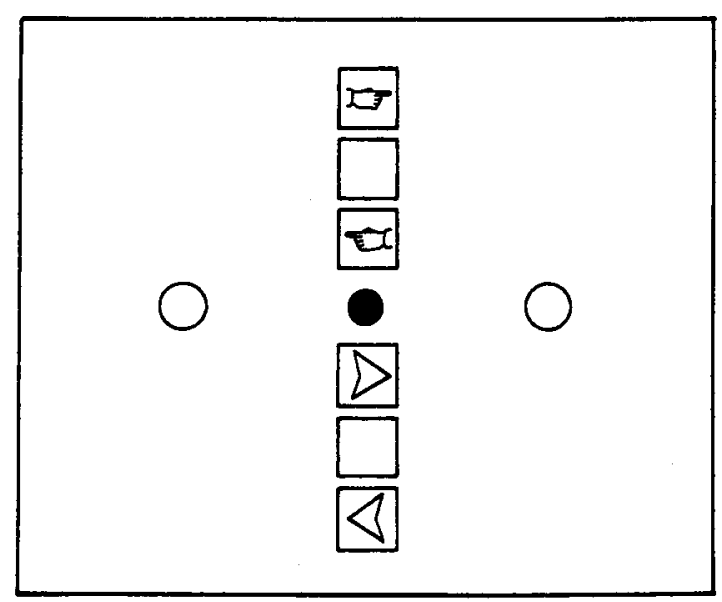

Figure 9. Schematic diagram of stimulus display in Verfaellie, Bowers, and Heilman's (1988) experiment. Target stimuli were circles of one of two brightnesses that occurred to the left or right. One of the three boxes above fixation was lit as the response-location precue, and one of the three boxes below fixation was lit as the stimulus-location precue. From "Attentional Factors in the Occurrence of StimulusResponse Compatibility Effects," by M. Verfaellie, D. Bowers, \& K. M. Heilman, 1988, Neunopsychologia, 26, 435-444, Figure 1. Copyright 1988 by Elsevier Science Ltd. Reprinted by permission. 
Proctor et al. (1992) also examined simpler procedures in which the stimulus- and response-location precues were manipulated in separate experiments. Still, the magnitude of the Simon effect was affected by only the response-location precues. In addition, response-location precuing was shown to enhance the magnitude of the Simon effect even when two stimuli were assigned to each response, ruling out the possibility that subjects were preparing for a specific stimulus instead of the cued response. The enhancement by the response-location precue was also shown to occur regardless of whether the hands were crossed or uncrossed. On the whole, the results obtained by Verfaellie et al. (1988) and Proctor et al. suggest that centrally presented precues influence the magnitude of the Simon effect only when an expectancy for a particular response is created. In other words, spatial coding is enhanced by an expectancy to respond at a particular location but is not affected by an expectancy for the particular location in which the target stimulus will occur. Such results seem easy to reconcile with response-selection accounts of the Simon effect but are problematic for stimulus-identification accounts.

In Proctor et al.'s (1992) experiments, there was a tendency for a reversed Simon effect to be obtained on trials for which an informative response-location precue was invalid. That is, responses on invalid trials tended to be faster when the target stimulus occurred in the location that corresponded with that of the precued response rather than with that of the actual response. Hasbroucq and Possamai (1994) focused on this difference between valid and invalid trials to argue that Proctor et al.'s results really show precuing of stimulus location, rather than response location, to be crucial and provide unique support for Hasbroucq and Guiard's (1991) stimulus-identification account of the Simon effect, which we described earlier. Hasbroucq and Possamai based their argument on tables that specified, separately for the valid and invalid precue trials in each experiment, the correspondence relations (+ for corresponding, and - for noncorresponding) among the four variables of precued location (left or right), stimulus meaning (whether the relevant stimulus information, e.g., bright or dim stimulus, indicated a left or right response), stimulus location (left or right), and response location (left or right). To determine which of these relations might be crucial to the obtained results, Hasbroucq and Possamai compared the correspondence relations between the valid and invalid trials.

For informative response-location precues, Hasbroucq and Possamai (1994) noted that only the relation between precued location and stimulus location both differentiated the corresponding trials from the noncorresponding trials for valid precues and reversed for invalid precues. Consequently, they concluded that the influence of the informative response-location precues on the Simon effect "demonstrates that the magnitude of the Simon effect depends on irrelevant precuing of stimulus location" (Hasbroucq \& Possamai, 1994, p. 238). Similarly, for informative stimulus-location precues, Hasbroucq and Possamai noted that two relationships, those between pre- cued location and response location and between precued location and stimulus meaning, differentiated the corresponding trials from the noncorresponding trials for valid precues and reversed for invalid precues. Because the informative stimulus-location precues had no influence on the Simon effect, they concluded that "the Simon effect is influenced neither by the irrelevant precuing of the location of the response nor the meaning of the stimulus" (Hasbroucq \& Possamai, 1994, pp. 239-240).

Hasbroucq and Possamai (1994) went on to reason that if irrelevant precuing of stimulus location influences the Simon effect but irrelevant precuing of response location does not, then "the evidence gathered by Proctor et al. is exclusively compatible with the hypothesis according to which the Simon effect reflects the processes involved in stimulus identification" (p. 244). The logic that Hasbroucq and Possamai use to arrive at this conclusion seems convoluted to us. The major problem, as we see it, is that their analysis is based on an atheoretical cataloging of the binary correspondence relations among the variables that ignores the fact that some of these relations are defined by the task as relevant and others as irrelevant. Both Verfaellie et al.'s (1988) and Proctor et al.'s (1992) results consistently show the Simon effect to be influenced by centrally presented precues that are informative about the likely response location. This influence would seemingly have to arise from processes that involve using the response information. When you add in the fact that explicitly precuing stimulus location in a similar manner has no influence on the Simon effect, the argument that the precuing results support stimulus-identification accounts of the Simon effect does not seem sustainable.

Hommel (1993a) compared the effects of central stimulus-location precues, like those used by Verfaellie et al. (1988) and Proctor et al. (1992), with those of peripherally presented precues and found them to be different. In his experiment, subjects saw two boxes, one to the left and one to the right of fixation, that designated the locations in which a color target stimulus could occur. Precues could be either arrows at a central location pointing to the left or right or brief flashes of the left or right box at one of the peripheral stimulus locations. A precue occurred 100 or $1,000 \mathrm{msec}$ before the target onset and indicated the target location with $80 \%$ probability. The central precues had no influence on the magnitude of the Simon effect, as in the studies of Verfaellie et al. and Proctor et al.: The Simon effect was approximately $25 \mathrm{msec}$ when the target occurred at the cued location and $28 \mathrm{msec}$ when it occurred at the uncued location. In contrast, with the peripheral precues, the Simon effect was smaller when the target occurred at the cued location (approximately $15 \mathrm{msec}$ ) rather than the uncued location (approximately $35 \mathrm{msec}$ ).

According to the attention shifting account of the Simon effect, if attention is shifted to the target location prior to onset of the target, target location should not be coded and the Simon effect should be reduced or eliminated. One interpretation of Hommel's stimulus-location 
precuing results therefore would be that attention shifts to the target location when the precue is presented peripherally but not when it is presented centrally. The problem with such an interpretation is that it requires the ad hoc assumption that subjects do not shift attention to the precued target location when a central precue is used, even though the precue is highly valid. Moreover, aspects of Hommel's (1993a) results are difficult to reconcile with this assumption. For one, the central precues had no greater influence on the Simon effect in his study when the SOA was $1,000 \mathrm{msec}$, which is sufficient time to allow for a voluntary shift of attention, than when it was $100 \mathrm{msec}$, which likely is not. For another, the overall decrease in RT produced by the central precues, which did not reduce the Simon effect, was similar in magnitude to that produced by the peripheral precues, which did reduce the effect.

Hommel (1993a) proposed an alternative account of his stimulus-location precuing results in terms of the temporal overlap hypothesis. According to this explanation, the peripheral precue produces a spatial code that then inhibits the formation of a spatial code for the target when it occurs at the precued location. The primary drawback for this account is that arrow stimuli produce evidence for spatial coding similar to that produced by physical location stimuli in many other cases (e.g., Lu \& Proctor, 1994; Weeks \& Proctor, 1990). Thus, it is unclear why the central precues would not also produce a spatial code that would then inhibit the formation of a spatial code for the target.

The finding that central stimulus-location precues do not influence the magnitude of the Simon effect is contradicted by an experiment of Stoffer and Yakin (1994). The general intent of their experiment was the same as that of Hommel's (1993a), with left and right pointing arrows used as precues, but the specific procedure differed in several ways: Precues were of $100 \%$ validity, with $22.2 \%$ of the trials being catch trials for which a stimulus presented prior to the precue indicated that a response was not to be made; the target stimulus was either a rectangle or a triangle rather than colors, and the SOA between the precue and target onsets was 50,500, or $700 \mathrm{msec}$. In contrast to Hommel's findings, Stoffer and Yakin found that the Simon effect decreased from approximately $25 \mathrm{msec}$ at the $50-\mathrm{msec}$ SOA to only $3 \mathrm{msec}$ at the 700-msec SOA. In agreement with Hommel's findings, in another experiment, peripheral precues were shown to produce a similar reduction in the magnitude of the Simon effect.

The reduction of the Simon effect obtained by Stoffer and Yakin (1994) with a central stimulus-location precue poses problems for Hommel's (1993a) account of his results in terms of inhibition of spatial coding, because this inhibition should only be present if the precue occurred peripherally at the target location. The attention shifting account can accommodate all of the findings for central and peripheral precues if it is assumed that discriminations of brightness or color do not require a shift of focused attention and that, consequently, atten- tion is not shifted when central precues are used for tasks involving such discriminations. The primary problem for this explanation is that, as noted previously, Hommel found overall precuing benefits of similar magnitude for central and peripheral precues, but with no reduction of the Simon effect for the central precue, when the two possible targets differed in color. Thus, attention appears to have been shifted to the target location in his study even when central precues were used, suggesting that the Simon effect should have been reduced.

To summarize, the results of the precuing studies show that the magnitude of the Simon effect is (1) not influenced in many cases by a valid central stimulus-location precue (Hommel, 1993a; Proctor et al., 1992; Verfaellie et al., 1988; with Stoffer \& Yakin, 1994, being an exception), (2) decreased by a valid peripheral stimuluslocation precue (Hommel, 1993a; Stoffer \& Yakin, 1994), and (3) increased by a central response-location precue (Proctor et al., 1992; Verfaellie et al., 1988). A shortcoming of the attention shifting and temporal overlap explanations that have been developed to explain the effects of stimulus-location precues is that they do not consider response expectancy effects. Yet, the enhancement of the Simon effect by informative response-location precues implies that expectancy to respond at a particular location is important. A complete account of the influence of precuing on the Simon effect will have to explain the effects of both stimulus-location and response-location precues.

\section{Summary}

The Simon effect is a widespread and persistent phenomenon that is attributed by most authors to responseselection processes. The effect apparently arises from stimulus location being coded even though this location information is defined as irrelevant to the task. It reflects more than just a tendency for the performer to respond to the side on which the stimulus occurs. The frame of reference with respect to which spatial coding of both the stimuli and the responses occurs is not fixed in relation to the observer but varies; in many cases, spatial coding is a function of relative position. Moreover, the direction of the Simon effect is reversed when the relevant stimulus dimension is mapped incompatibly to responses in the Hedge and Marsh task variation or when the task is defined in terms of an action goal (e.g., turning on a light) that is mapped incompatibly to the response key that controls that action.

The two alternative accounts for how and when a spatial code will be produced that have received the most support are referential coding with respect to objects or frames of reference in the display and shifting of attention from a prior location to the target location. Several results have suggested that attention shifting may play an important role, but the findings are far from unequivocal. For example, the evidence regarding a predicted decrease in the magnitude of the Simon effect from precuing stimulus location is conflicting. The evidence for the referential coding account is also mixed, with smaller 
Simon effects sometimes obtained when an obvious referent object is present than when one is not. Also, both the attention shifting and the referential accounts have no means for explaining the finding that the Simon effect is increased in magnitude when the likely response is precued. More principled explanations about factors that influence the direction and shifting of attention and the adoption of reference frames must be developed before this issue can be resolved.

Most accounts of the Simon effect have focused on factors determining whether the spatial code for the irrelevant location information is formed. A common assumption seems to be that if a spatial code is formed, then the Simon effect will be present. As a general rule, there has been little detailed consideration of how the strength and time course of the activation of this irrelevant information in relation to that of the relevant information influences the Simon effect. Yet, there is evidence to indicate that factors such as the relative processing speeds of the irrelevant and relevant information influence the magnitude of the Simon effect. Because the effect magnitude is apparently influenced not only by whether a spatial code is formed but also by its timing and relative strength with respect to the activated relevant information, complete models of the Simon effect will need to take into consideration this dynamic interaction. At present, Hommel's (1993b) temporal overlap account and De Jong et al.'s (1994) two-component model are the only ones that do so.

\section{THE SPATIAL STROOP EFFECT}

\section{The Basic Phenomenon}

The relevant and irrelevant stimulus dimensions used in the Stroop task are either identical or very similar in meaning. One dimension usually is a word, and the other is a physical attribute. In the original Stroop task (Stroop, 1935/1992), the two stimulus dimensions are a color word and the physical color in which it is printed. A condition in which the color word is incongruent with the physical color (e.g., the word RED in the color green) is compared with a baseline condition (e.g., a patch of green color) or to a condition in which the color word and physical color are congruent (e.g., the word RED in the color red). The standard Stroop effect is that colornaming responses are considerably slower for the incongruent condition than for either of the other two conditions. In contrast, word reading shows little influence of being printed in an incongruent color.

By including both the congruent and the baseline conditions in a study, it is possible to evaluate whether the Stroop effect is due primarily to inhibition on incongruent trials or facilitation on congruent trials. In a study by Glaser and Glaser (1982), the stimuli were four German color words-rot, gelb, grün, and blau (i.e., red, yellow, green, and blue) - and their respective colors. For the color-naming task, the neutral control consisted of strings of uppercase versions of the letter I in the different colors. When both dimensions of the stimulus were pre- sented simultaneously, there was a slight (16-msec) facilitation for naming the color of a congruent stimulus relative to the control condition, but a large $(72-\mathrm{msec})$ interference effect for naming an incongruent stimulus. Although there are many issues involved in interpreting differences with respect to a supposed baseline condition (Jonides \& Mack, 1984), this pattern of results is fairly typical. Glaser and Glaser noted that the interference from incongruent dimensions reported by most studies ranges from $70 \mathrm{msec}$ up to $200 \mathrm{msec}$, and it is generally accepted that the magnitude of facilitation from congruent dimensions is much less (MacLeod, 1991).

Spatial variations of the Stroop task have been used to examine the influence of irrelevant spatial information. The spatial Stroop tasks use verbal or symbolic stimuli, combining a semantic attribute that designates spatial location or direction with a physical stimulus position attribute. Either attribute can be defined as relevant or irrelevant for determining the correct response, which is made manually or vocally. By manipulating the semantic and physical position variables, the spatial Stroop stimulus can be presented in the position congruent or incongruent with the meaning signified by the semantic attribute.

As in the color Stroop task, incongruent location words produce interference in the spatial Stroop task. For example, White (1969) used a procedure in which a stimulus was presented inside of a square, at the top, bottom, right, or left, signifying the directions north, south, east, and west, respectively. Subjects responded to the location in which the stimulus appeared by saying the appropriate direction name or moving a lever in one of four corresponding directions. In an incongruent condition, the stimulus was a direction word (NORTH, SOUTH, EAST, or WEST) that was incongruent with the word's position, whereas in the control condition, the stimulus was a row of asterisks. Interference scores were computed by taking a ratio of the time to respond to a list of 80 items for the incongruent condition with respect to the time to respond to a list of the same length in the control condition. The interference score was 1.2 for the naming responses but close to 1.0 for the manual responses, indicating that an incongruent word slowed vocal responses to stimulus location but not manual responses.

Dyer (1972, Experiment 2) included the conditions necessary to evaluate whether facilitatory and inhibitory components contribute to the Stroop effect that occurs when physical location must be named. The stimuli for the experimental conditions in his study were the location names UP, DOWN, RIGHT, and LEFT, whereas the stimulus for the control condition was Xxxx. At the beginning of each trial, a stimulus was presented at the center of the display. It then moved in one of four directions (up, down, right, or left), and subjects were to name the direction in which the stimulus moved. Both inhibition $(14 \mathrm{msec})$ and facilitation $(26 \mathrm{msec})$ were found. Although facilitation was larger than inhibition in this study, this pattern apparently is unique to the movement stimuli used by Dyer. For example, a study by Shor (1970), 
described later in this section, showed substantial interference and little facilitation when the nonverbal stimulus dimension was the direction in which an arrow pointed. In general, the spatial Stroop effect seems to reflect primarily interference when the irrelevant dimension is incongruent with the relevant dimension, but the interference is less than for the Stroop color-naming task (Clark \& Brownell, 1975; Dalrymple-Alford, 1972; Dyer, 1973, 1974; MacLeod, 1991; Shor, 1970).

The spatial Stroop effect has been demonstrated for many other task variations in which the relevant and irrelevant attributes are integrated within a single stimulus, as in White's (1969) and Dyer's (1972) studies described above. For example, Harvey (1984) had subjects make "same"-"different" keypress responses regarding whether the position (high or low) of a neutral first stimulus matched the position (high or low) or symbolic content of a two-letter word (HI or LO). Correct "same" responses to the word content were $71 \mathrm{msec}$ slower on average when the location in which the word occurred was incongruent with the symbolic information than when it was congruent. Clark and Brownell (1975) instructed subjects to judge whether an arrow presented inside a rectangle was pointing up or down by making a left or right keypress response. The arrow could occur at any of six positions along the vertical midline of the rectangle, three of which were above center and three of which were below. Responses averaged $42 \mathrm{msec}$ faster when the location of the arrow with respect to center corresponded with the direction in which it pointed than when it did not, with the magnitude of this effect being an increasing function of the distance from center.

The spatial Stroop effect has also been obtained for more complex displays in which all elements are not integrated within a single stimulus. Seymour (1973) used stimulus displays in which both a location word (ABOVE, BELOW, LEFT, or RIGHT) and a dot were presented simultaneously. Subjects were asked to name the position of the dot relative to the word or the location of the word relative to the dot. The meaning of the word had little effect on the time to name the dot location, but it had a significant effect on the time to name the location occupied by the word. Responses to the word's location were particularly slow when the word specified the opposing location on the same dimension (e.g., the word LEFT in the right location).

Palef (1978) conducted similar experiments, presenting the words ABOVE and BELOW directly above or below an asterisk (relative position) and either above or below the midline of the display screen (absolute position). Subjects were required to respond to the meaning of the word, its absolute position, or its relative position by pressing one of two response keys, which were located one beside the other and labeled above and below. When absolute position was relevant, neither word meaning nor relative position affected performance. Similarly, when word meaning was relevant, there was also no significant effect of absolute or relative position. However, when relative position was relevant, the irrelevant word meaning and absolute position produced additive Stroop effects. According to Palef, the relative position is first coded pictorially and then translated into a linguistic code for the response. The effects of absolute position and word meaning on responding to relative position are additive because absolute position influences the pictorial coding stage and word meaning influences the linguistic coding stage.

Finally, Shor (1970) conducted experiments using a spatial and pictorial analog to the standard Stroop task in which the two stimulus dimensions were not integrated. The direction name UP, DOWN, LEFT, or RIGHT was embedded within an outline drawing of an arrow pointing in one of the four directions. Naming the direction in which the arrow pointed was slower than naming the direction word. Moreover, naming the direction of the arrow was slowed when the direction word was incongruent, but naming the direction word was not slowed by an incongruent arrow direction. Shor, Hatch, Hudson, Landrigan, and Shaffer (1972) showed that the spatial Stroop effect for naming the arrow direction persisted even after extended practice. Although RTs decreased considerably across 30 days of practice, naming whether an arrow pointed left or right was still slower than reading the location name, and there was little or no reduction in the magnitude of the Stroop effect (the effect was approximately $45 \mathrm{msec}$ for the first five sessions and $35 \mathrm{msec}$ for the last five sessions).

The studies just reviewed indicate that spatial Stroop effects can be obtained for both integrated and nonintegrated stimuli. However, MacLeod (1991) has suggested that this does not guarantee that effects for integrated and nonintegrated stimuli are due to the same mechanism. MacLeod's suggestion is based on a study by Flowers and Stoup (1977) using color stimuli in which the influence of practice on the Stroop effect for integrated and nonintegrated stimuli was compared in a card-sorting task. The integrated stimuli were color names printed in different ink colors (e.g., the word RED printed in green ink), whereas the nonintegrated stimuli were color names presented inside of color-outlined rectangles (e.g., the word RED inside a green-outlined rectangle). Flowers and Stoup found that the Stroop effect for integrated stimuli persisted over four practice sessions, whereas that for nonintegrated stimuli did not.

No similar comparison has been performed for the spatial Stroop task, so it is not known whether the effect of practice would be different for integrated and nonintegrated stimuli. However, as mentioned previously, Shor et al. (1972) demonstrated that the spatial Stroop effect obtained with nonintegrated stimuli persisted throughout 30 days of practice. This result suggests that the Stroop effect obtained with nonintegrated spatial Stroop stimuli is not as transient as that obtained with nonintegrated color Stroop stimuli in the card-sorting procedure used by Flowers and Stoup (1977).

\section{Processing Stage at Which the Effect Occurs}

Most explanations of both the color and the spatial Stroop effects attribute them to competition at the stage 
of response selection (Dyer, 1973). The two dimensions of the Stroop stimulus are presumed to be processed in a parallel manner, with a response code activated for each (e.g., Glaser \& Dolt, 1977; Keele, 1972; Morton \& Chambers, 1973; Posner \& Snyder, 1975; Warren, 1972). When both stimulus dimensions activate the same response code, no conflict occurs and responding may be facilitated by the redundancy. However, if the response code for the irrelevant dimension is incongruent with that for the relevant dimension, the irrelevant response code must be suppressed before the response can be executed.

As would be expected if the spatial Stroop effect were a response-selection phenomenon, many of the results obtained with the spatial Stroop task are similar to those obtained with the Simon task. For example, when responding to the location word with a keypress, a substantial effect of its location is evident even when an uninformative stimulus occurs in the location opposite that of the target stimulus (O'Leary \& Barber, 1993). Also, as in the Hedge and Marsh variation of the Simon task, an alternate-location mapping (i.e., a left keypress response is made to the word RIGHT and a right keypress response to the word LEFT) produces a reversed effect of faster responding when the stimulus occurs in the location opposite the response than when it occurs in the corresponding location (Arend \& Wandmacher, 1987; Lu \& Proctor, 1994).

Only a few studies are in disagreement with the response competition account, with the most notable being that of Hock and Egeth (1970). They used a memory search task in which subjects had to indicate whether the color of a target stimulus was included in a memory set by responding "yes" or "no." A Stroop effect was obtained, with responses being slower when the form in which the color was printed spelled an incongruent color word than when it was a series of Xs. This effect did not interact with the size of the memory set, suggesting that it was not due to processes involved in comparing the target to the memory set. Moreover, the verbal responses of "yes" and "no" can be assumed to have no direct semantic relation to the printed words, suggesting little opportunity for competition in the selection of overt responses. Consequently, Hock and Egeth concluded that the Stroop effect took place at the stage of stimulus identification.

However, Dalrymple-Alford and Azkoul (1972) pointed out that Hock and Egeth's (1970) results do not rule out response competition based on covert naming responses. Dalrymple-Alford and Azkoul noted that, because the memory set was defined in terms of color names, the target color would likely have had to have been named covertly for comparison with the memory set. Response competition effects that would delay generation of this covert naming response would precede comparison with the memory set and, therefore, would not be expected to interact with memory-set size. Thus, although Hock and Egeth's results suggest that competition between overt responses is not necessary for the Stroop effect to occur, they do not rule out response competition in general as the basis for the Stroop effect. In fact, one of the authors, Egeth, has more recently advocated a response competition account in his work with Virzi (Virzi \& Egeth, 1985), which we discuss later in the paper.

The S-S congruity account proposed by Hasbroucq and Guiard (1991) for the Simon effect, which attributes the effect to stimulus-identification processes, also is applicable to the spatial Stroop effect. O'Leary and Barber (1994) evaluated Hasbroucq and Guiard's account by having subjects judge whether a location word (LEFT or RIGHT) and the location in which it was presented (left or right) were congruent or incongruent. This procedure allowed them to dissociate S-S congruity from the correspondence relations between the irrelevant stimulus dimensions and the response location. The results showed a 45-msec advantage of S-S congruity, but this is not unusual since it is well established that judgments of congruence, or sameness, are typically faster in such situations than are judgments of incongruence, or difference (e.g., Proctor, 1981). Although this effect might possibly reflect interference in stimulus identification of the type proposed by Hasbroucq and Guiard, it is not strong evidence for such interference because (1) neither stimulus dimension was irrelevant, as in the situations for which Hasbroucq and Guiard's account was proposed, and (2) interference has typically been assigned at most a secondary role in the "fast-same" effect, regardless of the processing stage to which the interference is attributed. The most important finding in O'Leary and Barber's study was that both irrelevant spatial S-R correspondence (i.e., the relation between stimulus location and response location) and irrelevant verbospatial $\mathbf{S}-\mathbf{R}$ correspondence (i.e., the relation between the location word and response location) had substantial effects of 62 and $116 \mathrm{msec}$, respectively. Thus, with spatial Stroop stimuli, strong effects of the correspondence between each stimulus dimension and the response location are obtained when they are dissociated from any effect of S-S congruity.

\section{Asymmetrical Effects and the Horse Race Model}

In the original color Stroop task, an incongruent color word affects the time to name the physical color, but an incongruent physical color does not affect the time to read the color word. A similar asymmetry has been shown in the spatial Stroop task. Palef and Olson (1975) instructed subjects to respond to the meaning or position of the words ABOVE and BELOW presented either above or below an asterisk, by making an "above" or "below" keypress. In Experiment 1, subjects were seated facing the screen at a distance of $2 \mathrm{ft}$. The height of the word stimuli was approximately $2^{\circ}$ of visual angle, and the space between the word and the asterisk was about $2^{\circ}$. Position information was processed faster than was word meaning; more importantly, position affected responding to word meaning, but not vice versa.

In Palef and Olson's (1975) Experiment 2, the reactions to the spatial position of the word were slowed by some modifications. The distance from which subjects viewed the screen was increased to $6 \mathrm{ft}$, decreasing the 
height of the word and the space between the word and the asterisk to approximately $1^{\circ}$ of visual angle. Also, whereas in Experiment 1 the spatial locations of the words were above or below both with respect to an asterisk (relative position) and in terms of absolute position on the screen, in Experiment 2 only relative position was varied. Palef and Olson assumed that reactions to spatial position of the word would be longer for this stimulus display. The results showed that word meaning was processed faster than was spatial position, although this difference was overcome with practice. The fast word processing affected the slower position processing in the first session of trials. Moreover, when the relative processing times of position and word meaning were equal in the second session of trials, the Stroop effect was bidirectional.

To explain their results, Palef and Olson (1975) suggested that the occurrence of the Stroop effect "depends on the relative speeds with which the two dimensions of information are processed" (p. 201). For the standard color Stroop task, the faster word processing always affects the slower color processing, whereas the color processing does not affect the word processing. According to Palef and Olson's "empirical rule," a Stroop effect can occur only when the irrelevant dimension is available before a decision is made about the relevant dimension.

A horse race model of interference, deriving from the relative speed hypothesis, has been suggested by many researchers (e.g., Klein, 1964; Morton \& Chambers, 1973; Palef \& Olson, 1975; Posner, 1978; Posner \& Snyder, 1975; Stroop, 1935/1992). It presumes that the processing of the relevant and irrelevant stimulus dimensions occurs in parallel prior to a response buffer, which is a single centralized response channel. Interference arises in the response buffer when the incorrect response signified by the irrelevant information is entered into the buffer before the correct response and needs to be overcome. A specific account of this type is provided by Morton and Chambers's (1973) logogen model, in which multiple stimuli enter in parallel into a logogen system (a pattern recognition system with an internal recognition unit for each possible stimulus) that automatically produces appropriate internal responses to the stimuli. When more than one internal response is activated, they compete for entry into a single channel processor for production of the overt response. Because the internal response that is activated first has priority for entry into the single channel processor, the relative speed at which the relevant and irrelevant information is processed determines whether Stroop interference will occur.

The horse race model is closely akin to Hommel's (1993c) temporal overlap account for the Simon effect, with two major exceptions. First, whereas Hommel's account explicitly includes the assumption that the activation produced by the irrelevant information decays, the horse race model does not. Second, and more importantly, whereas Hommel's account is restricted to the influence of irrelevant information about stimulus location on manual responses to symbolic stimulus information, the horse race model is intended to apply more generally to situations in which any two stimulus dimensions provide conflicting information.

\section{Evidence Against the Horse Race Model}

In the horse race model, the relative speeds with which the two stimulus dimensions are processed, independent of the specific response mode, are assumed to determine which dimension, when irrelevant, produces interference with responding to the other dimension. This assumption has been challenged by several empirical findings. Greenwald (1972) specified a relation between stimuli and responses of "ideomotor compatibility," based on the view that responses are coded by representations of their sensory feedback (e.g., James, 1890/1950). Ideomotor compatibility occurs when the stimulus resembles sensory feedback from its required response - for example, when a vocal naming response is required to an auditory verbal stimulus. Greenwald conducted experiments in which subjects had to make rapid independent responses to two simultaneous stimuli. An arrow pointing to the left or right was displayed visually, while an auditory stimulus, the word LEFT or RIGHT, was presented simultaneously through headphones. In the low-ideomotorcompatible condition, subjects were to move a switch left or right in response to the word LEFT or RIGHT, respectively, and to say "left" or "right" to the direction in which the arrow pointed. In the high-ideomotor-compatible condition, subjects were to say "left" or "right" to the auditory word stimulus and to move the switch left or right to the arrow direction. Greenwald found that reactions were slower in the low-ideomotor-compatibility condition than in the high-ideomotor-compatibility condition. Also, verbal responses were slower than manual responses, and RTs were increased when the two responses conflicted.

The ideomotor-compatibility findings are consistent with a study by McClain (1983) in which response modality was manipulated. In her study, the word HIGH or LOW was presented auditorily in either a high or a low pitch. McClain compared the time to identify the word with that to identify the pitch using three response modes: verbal (responding "high" or "low" vocally), keypress (pressing one of two keys labeled high or low), and hum (humming a high or low pitch). Large Stroop effects occurred when the relevant stimulus dimension and the response mode were of low ideomotor compatibility (i.e., pitch-verbal, pitch-keypress, word-hum), but not when they were of high ideomotor compatibility (i.e., wordverbal, word-keypress, pitch-hum). This finding suggests that the response modes are crucial to the Stroop effect.

Results obtained with visual spatial Stroop stimuli by Virzi and Egeth (1985) are in agreement with McClain's (1983) findings. In their Experiment 2, the location word LEFT or RIGHT was presented in the left or right hemifield. A Stroop effect was found when subjects made a vocal response ("left" or "right") to the position of the location word or a keypress response to the word's meaning. However, only small, nonsignificant Stroop effects were evident for keypress responses to position and vo- 
cal responses to word meaning. Shimamura (1987) examined only vocal or keypress responses to location and obtained results similar to those of Virzi and Egeth with irrelevant location words (1) in English for English readers and (2) in Japanese Kanja (which is a phonetic orthography, as is English) for Japanese readers. In contrast to the findings of these studies, the response mode would not be important if the Stroop effect were solely a function of a horse race between the two stimulus dimensions.

According to the horse race model, preexposure of the irrelevant dimension should speed up its identification and, consequently, its entry into the single response channel (e.g., Morton \& Chambers, 1973). When the irrelevant information already "wins the race" on at least some trials, thus producing a Stroop effect, no clear prediction can be derived from the race model: If the response activation does not decay, then the magnitude of the Stroop effect should, if anything, be increased by preexposure; however, if it is presumed that the response activation decays, as Hommel (1993c) does, then the Stroop effect should decrease at longer preexposures. In fact, this latter result is what is typically obtained.

With a standard color-naming Stroop task, Dyer (1971) presented the irrelevant word in black at one of several intervals before presenting the same word printed in color. The Stroop effect was $90,118,68$, and $32 \mathrm{msec}$ at the 0-, 40-, 200-, and 500-msec SOAs, respectively, indicating that the effect magnitude was a decreasing function of SOA. Glaser and Glaser (1982, Experiment 1) also found the Stroop color-naming effect to be a decreasing function of the SOA between the irrelevant and relevant stimulus information. When the irrelevant word was presented $0,100,200,300$, or $400 \mathrm{msec}$ before the relevant ink color, significant Stroop effects of 88,75 , 78,64 , and $56 \mathrm{msec}$, respectively, were evident. Lu and Proctor (1995) found a similar, though greatly attenuated, pattern of results for the spatial Stroop effect when responding with keypresses to location words (LEFT or RIGHT) embedded in irrelevant arrow stimuli that pointed to the left or right: Spatial Stroop effects of 25, 23, 18, 15,16 , and 18 msec occurred when the arrow onset preceded the word at SOAs of $0,100,200,300,400$, and $500 \mathrm{msec}$, respectively.

The horse race model makes a stronger prediction for situations in which the irrelevant information normally "loses the race" and, hence, produces no Stroop effect. That prediction is that preexposure of the irrelevant information in these cases should introduce a Stroop effect of sizable magnitude. However, this does not seem to occur. In contrast to their results obtained in the colornaming task, Glaser and Glaser (1982) found that the Stroop effect was never very large when the color of the stimulus was preexposed in a word-reading task (effects of $-2,3,12,7$, and $1 \mathrm{msec}$ at the $0-, 100-, 200-, 300-$, and 400-msec SOAs). Although there tended to be a small Stroop effect at the intermediate SOAs, the magnitude did not begin to approach that obtained for the color-naming task. Sugg and McDonald (1994) found similar asymmetries in a task for which the responses to Stroop color stimuli were manual aimed movements to locations on a touch screen labeled by either physical colors or color words. Substantial Stroop effects were apparent at SOAs of up to $300 \mathrm{msec}$ when word was the relevant stimulus dimension and the response labels were colors or when color was the relevant stimulus dimension and the response labels were words. However, only much smaller effects were present when word was the relevant stimulus dimension and the response labels were words or when color was the relevant stimulus dimension and the response labels were colors.

Dunbar and MacLeod (1984) applied logic analogous to that of the SOA procedure by slowing the word-reading latencies in the color Stroop task. In four experiments, word-reading latency was prolonged by using geometrically transformed words, along with manipulations of uncertainty about the word's orientation and the number of noncolor words to increase reading latency even further. According to the horse race model, slowing the faster process of word reading should decrease the effect of word meaning on the color-naming response. However, in all of Dunbar and MacLeod's experiments, the standard Stroop effect from incongruent words on the color-naming responses persisted unaltered by the degree of word-reading difficulty. When word reading was extremely slow, a reversed Stroop effect occurred in which an incongruent stimulus color interfered with word reading; but even in this case, the standard Stroop effect for color naming was also present, contrary to the prediction of the race model.

Evidence inconsistent with the predicted reversal of the asymmetry has also been obtained in the spatial Stroop task using the SOA procedure. In Virzi and Egeth's (1985) Experiment 3, the irrelevant dimension was preexposed at various SOAs before the relevant dimension for the manual-response-to-position and the vocalresponse-to-word-meaning conditions of the spatial Stroop task, which generally show little or no Stroop effect in the standard task design. In the manual-responseto-position condition, the preexposed stimulus was either the letter $\mathrm{R}$ or the letter $\mathrm{L}$ presented in the center of the visual field. The preexposed letter was always consistent with the initial letter of the target stimulus (i.e., the word RIGHT or LEFT) and thus specified the irrelevant stimulus dimension of word meaning. In the vocalresponse-to-word meaning condition, a bar was preexposed to one side or the other of the central fixation to specify the irrelevant stimulus location in advance of the location word onset. In both conditions, preexposing the irrelevant information did not yield any reliable Stroop effect $(5,2,3$, and $5 \mathrm{msec}$ for manual-response-to-position condition, and 4, 10,5, and $2 \mathrm{msec}$ for vocal-responseto-word-meaning condition for SOAs of 17, 50, 150, and $500 \mathrm{msec}$, respectively). Thus, contrary to the prediction of the horse race model, Virzi and Egeth found no evidence that preexposing the irrelevant information introduces a Stroop effect of sizable magnitude for conditions that do not normally show one. 


\section{A Translational Model}

Virzi and Egeth (1985) proposed that whether the Stroop effect occurs is a function of whether the relevant information is transmitted from encoding to response output via a translational mechanism. In their translational model (see Figure 10), several independent processing systems (e.g., the spatial system and the linguistic system in the spatial Stroop task) are presumed. It is hypothesized that the spatial and linguistic processing systems are linked by a translation mechanism. If the word meaning needs to be reported vocally (i.e., the vocal-response-to-word-meaning condition) or the word position needs to be indicated by keypresses (i.e., the manual-response-to-position condition), the encoding of the relevant stimulus information and the selection of the response are processed within the same system (i.e., the linguistic and spatial systems, respectively). According to Virzi and Egeth (1985), when the relevant information is processed within a single system and, hence, translation between systems is not required, no Stroop effect will occur. Glaser and Glaser (1989) developed a similar model that distinguishes between semantic and lexical systems. However, because they describe their model as "highly compatible" with Virzi and Egeth's and it was

TRANSLATIONAL MODEL

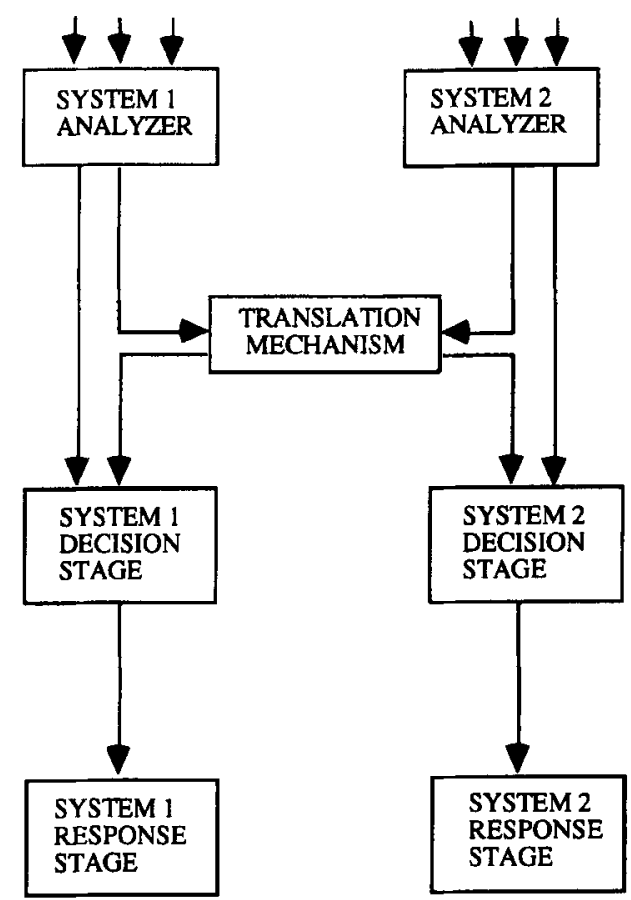

Figure 10. Virzi and Egeth's (1985) translational model. Two systems, for example, spatial-manual and verbal-vocal, are connected by a translation mechanism. A stimulus automatically activates a response code within the same system, but onty activates a response code in the other system when an intentional translation to that system is made. From "Toward a Translational Model of Stroop Interference," by R. A. Virzi \& H. E. Egeth, 1985, Memory \& Cognition, 13, 304-319, Figure 2. Copyright 1985 by the Psychonomic Society, Inc. Reprinted by permission. applied primarily to picture-word versions of the Stroop task, we will not consider it here.

In the translational model, when the position of a stimulus needs to be reported vocally (i.e., the vocal-responseto-position condition), the position information is encoded by the spatial system and then translated into the linguistic system for outputting the vocal response. Similarly, when the meaning of a word needs to be indicated by keypresses (i.e., the manual-response-to-word-meaning condition), the semantic information is encoded by the linguistic system and then translated into the spatial system for the output of keypress responses. In these situations, two response codes will be activated, one for the translated relevant stimulus dimension and one for the irrelevant stimulus dimension, for which translation is not necessary. The response codes generated by the relevant and irrelevant information are in competition at the decision stage of the system used to respond. The decision process will be slowed when these codes indicate different responses relative to when they indicate the same responses.

The translational model thus is in agreement with the horse race model in attributing the Stroop effect to competition between response codes activated by the relevant and irrelevant stimulus dimensions. The primary difference between the two models is that whereas the horse race model assumes a single response channel in which the activation of response codes is independent of response modality, the translational model assumes separate response channels for vocal and keypress responses, with a response code being activated for the irrelevant stimulus dimension only when it has a privileged access to the channel that is being used (i.e., irrelevant location information for keypress responses and irrelevant word information for vocal responses). The results of the SOA studies (e.g., Dyer, 1971; Glaser \& Glaser, 1982; Sugg \& McDonald, 1994) that are inconsistent with the horse race model can be explained by the translational model (Virzi \& Egeth, 1985). According to the translational model, for the word-reading task, it is not obligatory to process the irrelevant dimension of color information in the decision stage. Therefore, even when the color information is preexposed, it still would not be translated to the vocal response system, resulting in an absence of interference. However, for the color-naming task, it is obligatory to process the irrelevant word dimension in the decision stage because this dimension is in the linguistic system that is used for the response. Preexposing the irrelevant word information would cause a response code for it to be produced earlier, thus reducing competition with the response code generated when the relevant color information is subsequently presented.

The translational model predicts that the Stroop effect will be absent when the irrelevant information does not have privileged access to the channel used to respond to the relevant information (i.e., irrelevant word information for keypress responses and irrelevant location information for vocal responses). Yet, a Stroop effect of small magnitude appears to occur in these situations. O'Leary 
and Barber (1993) replicated the pattern of results reported by Virzi and Egeth (1985) for simultaneously presented verbal and nonverbal spatial information but found the Stroop effect in the vocal-response-to-wordmeaning condition of $11 \mathrm{msec}$ to be statistically reliable. Similarly, the keypress-response-to-location condition also tended to show a small Stroop effect in Virzi and Egeth's $(8 \mathrm{msec})$ and O'Leary and Barber's (7 msec) studies, although it was nonsignificant in both cases. We recently showed this effect to attain a significant magnitude of $18 \mathrm{msec}$ for keypresses made to left or right pointing arrows when the irrelevant location word was preexposed at an intermediate SOA of $300 \mathrm{msec}$ ( $\mathrm{Lu} \&$ Proctor, 1995). Apparently Virzi and Egeth missed this effect when they varied SOA, because they did not include SOAs between 150 and $500 \mathrm{msec}$. Because irrelevant location words influence keypress responses and irrelevant locations influence vocal responses, to at least some extent, translation of irrelevant information into activation of response codes is best viewed as varying in degree, as O'Leary and Barber have suggested, rather than being all-or-none.

\section{Automatic and Controlled Processing}

The distinction between controlled and automatic processes has been applied to the Stroop task (Posner \& Snyder, 1975). Automatic processes are fast and do not require attention for execution, whereas controlled processes are relatively slow and require attention (Shiffrin \& Schneider, 1977). Posner and Snyder (1975) assumed that, in a standard color Stroop task, the relatively fast word reading is automatic and the slower color naming is controlled. Their explanation can be applied to the spatial Stroop task in which incongruent location information interferes with manual responding to location name. The relatively fast location processing is considered to be automatic and the slower processing of word meaning is considered to be controlled. The basic idea is that the automatic process cannot be withheld and therefore can interfere with the controlled process, but not vice versa. Thus, the Stroop asymmetry can be explained easily in terms of automaticity.

Activation in semantic memory is often presumed to spread automatically from the identified concept to ones that are associated with it. Consistent with this assumption, a semantic gradient of interference is obtained for the Stroop effect. In the color-naming Stroop task, Klein (1964) examined conditions in which the words (1) were color names from a set different from the colors being named, (2) implicated specific colors (e.g., lemon), or (3) had no specific color meaning, in addition to a condition in which the irrelevant color words were from the set of colors being named. The results indicated that the more semantically related the irrelevant word is to the relevant color dimension, the more interference it causes. This finding has been replicated in numerous studies (e.g., Dalrymple-Alford, 1968, 1972; Proctor, 1978).

Similar findings of semantic similarity have been shown for the spatial Stroop effect. Fox, Shor, and Stein- man (1971) suggested that spatial Stroop interference is primarily a function of the semantic relationship to spatial direction. The stimulus in their experiment was a word or group of letters printed near the top, bottom, right, or left of a square. Subjects needed to ignore the word or letter group and to name the position (i.e., up, down, right, and left) of the stimulus in the square. The stimuli were printed in seven types of charts with different degrees of semantic gradient: (1) same direction names as the vocal response (i.e., UP, DOWN, RIGHT, LEFT) presented in incongruent position, (2) different direction names (i.e., NORTH, SOUTH, EAST, WEST), (3) direction-associated verbs (i.e., LIFT, DROP, TURN, FLOW), (4) common words (IT, GRAY, HEART, BOOK), (5) rare words (PI, DIRK, STOAT, TARN), and (6) consonant groups (i.e., JB, FWXD, TRTDR, RDLN), and (7) undifferentiated groups of four letter Os each. A logarithmic transformation of the RT data showed differences among the seven conditions, with the order of mean RTs from largest to smallest being Conditions $1,2,4,3,6,5$, and 7; all differences between nonadjacent mean RTs were significant. Fox et al. concluded that the amount of interference corresponded to a semantic gradient.

The semantic gradient in the Stroop task can be easily explained in terms of spreading activation and response competition. For example, if the two alternative locations to be named are left and right, the response "right" will be activated to a decreasing degree by the words RIGHT, EAST, and BOOK. When the correct response to the stimulus location is "left," the amount of response competition and, hence, of Stroop interference will be a decreasing function of the association of the word to the response "right."

Although automatic and controlled processes are usually treated as a dichotomy, there is evidence with the Stroop task to suggest that they should be viewed as points along a continuum. MacLeod and Dunbar (1988) demonstrated that the attributes of automaticity involved in the Stroop task develop gradually through practice. They had subjects practice responding with assigned color names to four novel shapes and examined the patterns of interference in color-naming and shape-naming variations of the Stroop task after various amounts of practice. Initially, shape naming was considerably slower than was color naming, and only the shape-naming task showed a Stroop effect. However, with practice, shape naming became as fast as color naming, and the pattern reversed such that only the color-naming task showed a Stroop effect. The reversal of the Stroop effect as the initially "nonautomatic" dimension is practiced and becomes "automatic" suggests that instead of automaticity being all-or-none, it is a continuum. This implication is in agreement with results showing that detection performance in visual search tasks is an increasing function of the degree of consistency with which a letter occurs as a target rather than as a distractor (e.g., Schneider \& Fisk, 1982).

\section{Allocation of Attention \\ Most Stroop studies have not examined the possible influence of attentional factors. Exceptions are several}


studies by Logan and Zbrodoff (1979, 1982; Logan, 1980; Logan, Zbrodoff, \& Fostey, 1983), who hypothesized that if the relation between congruent and incongruent dimensions were known, the task could be performed with different attentional strategies. One way to study these strategies is to manipulate the relative frequency of congruent and incongruent trials, and another is to precue the relation between dimensions at the beginning of each trial.

Logan and Zbrodoff (1979) used the first procedure and showed that the spatial Stroop effect can be reversed by increasing the relative frequency of the incongruent trials. Subjects were instructed to make a left or right keypress to either the identity or the position of a location word (ABOVE or BELOW) presented above or below a central fixation. At most, a small Stroop effect was observed with the keypress-to-position task, as has generally been found in other studies, and this effect was independent of the relative frequency of incongruent trials. However, the keypress-to-word task showed a crossover of the Stroop effect as a function of relative frequency. When the incongruent trials were relatively infrequent $(10 \%, 20 \%$, or $40 \%$ of the trials), responses to incongruent stimuli were significantly slower than were those to congruent stimuli. But when the incongruent trials were relatively frequent $(80 \%$ and $90 \%$ of the trials), responses were faster to the incongruent stimuli than to the congruent stimuli. According to Logan and Zbrodoff, the advantage for incongruent stimuli when they are relatively frequent suggests that subjects adopt a strategy of dividing attention between the relevant and irrelevant dimensions. The magnitude of attentional weight given to the irrelevant dimension would increase in proportion to the relative frequency of incongruent trials to optimize performance.

Logan (1980) used a procedure similar to the one used by Palef (1978) in which the word ABOVE or BELOW occurred above or below an asterisk, and the whole configuration appeared above or below a central fixation point. Subjects responded to the relative position of the asterisk and word by a left or right keypress. With this procedure, two stimulus dimensions, word identity and absolute position, are irrelevant. For the irrelevant dimension of absolute position, RTs were considerably slower for incongruent trials than for congruent trials when only $20 \%$ of the trials were incongruent, and this relation reversed when $80 \%$ of the trials were incongruent, similar to the relative frequency effect shown by Logan and Zbrodoff (1979). A similar interaction, but of reduced magnitude, was obtained for the irrelevant dimension of word identity. The probable reason why the validity of irrelevant word identity had an effect on keypress responses to position in Logan's study but not in Logan and Zbrodoff's is that responses were slowed considerably by the more complex procedure used by Logan.

Logan and Zbrodoff (1982) took the second approach described above of precuing the relation (congruent or incongruent) between the two stimulus dimensions on each trial. The task involved making keypress responses to the word location name, with a precue preceding onset of the Stroop stimulus at intervals ranging from 100 to $1,000 \mathrm{msec}$. The letter X always indicated that the Stroop stimulus would be congruent (e.g., the word ABOVE presented above the central fixation), whereas the letter $O$ always indicated that the Stroop stimulus would be incongruent (e.g., the word BELOW presented above the central fixation). A neutral cue control session was also conducted in which the cues $\mathrm{X}$ and $\mathrm{O}$ provide no information about the relation between the two dimensions of the Stroop stimulus. Logan and Zbrodoff hypothesized that subjects would construct different strategies for different cue validities and cue onset delays. Their Experiments 1 and 2 showed that validly precuing either congruent or incongruent stimuli reduced RTs, with the precuing benefits reaching asymptote when the interval between the onsets of the cue and Stroop stimuli was $600 \mathrm{msec}$. Moreover, the precuing benefit was much larger for congruent stimuli than for incongruent stimuli. Their Experiment 3 demonstrated that the primary change in this pattern of results that occurs as a function of practice is for the asymptotic benefit to be attained at progressively shorter precuing intervals.

The finding that it is more beneficial to provide advance information that the forthcoming stimulus is congruent rather than incongruent is similar to an asymmetry observed in experiments that have examined conditions in which compatible and incompatible $S-R$ mappings are mixed. In such experiments, compatible trials often show more benefit from being precued or presented in distinct blocks than do incompatible trials (e.g., Proctor, Lu, Wang, \& Dutta, in press; Stoffels, 1995; Van Duren $\&$ Sanders, 1988). The interpretation given by those authors for the fact that there is a unique advantage associated with knowing that all S-R relations are compatible is that automatic response tendencies must be inhibited unless they are known to be correct. Logan and Zbrodoff (1982) provided a similar interpretation for their situation in which the uncertainty involves the relation between irrelevant and relevant information, suggesting that "the strategic use of position information amplifies the automatic compatibility effects, as if attention to the word's position activated habitual associations between word identity and position more than they would have been activated had attention been restricted to word identity" (p. 519). Although stated in terms of activation instead of inhibition, the point is the same: There is a unique advantage associated with allowing the automatic response tendencies produced by irrelevant or relevant information free rein that is manifest when these tendencies are known to be correct.

Logan et al. (1983) used a dual-task procedure to evaluate more specifically the nature of the way in which the precued information was being used in strategy construction. They had subjects perform a secondary task of responding to a probe tone presented on half of the trials at varying delays after the start of the spatial Stroop task sequence, which was the same as in Logan and Zbrodoff (1982). When the response to the probe tone was a key- 
press, probe RTs were elevated substantially in valid cue trial blocks relative to neutral cue trial blocks at all but the longest probe delays. However, when the response to the probe tone was a vocal utterance (the syllable /ba/), probe RTs were no slower in valid cue blocks than in neutral cue blocks. These results are consistent with the distinction between manual and vocal responses made in Virzi and Egeth's (1985) model and were interpreted by Logan et al. as indicating that the strategy underlying the precuing benefits involves establishing a set of rules for mapping the stimulus locations onto the response locations.

To summarize, the studies of Logan and Zbrodoff leave no doubt that strategies play a significant role in the spatial Stroop task. The results of their experiments are generally consistent with a model proposed by Logan (1980) in which evidence from several sources is combined in a single decision process. In this model, performance is influenced by three major variables. First, the typical Stroop effect is attributed to automatic processing due to prior associations of the relevant and irrelevant information. Second, changes in the predictive relations between the two sources of information influence performance through causing different attentional strategies to be adopted. These strategies involve establishing rules for mapping stimuli onto responses by assigning weights to the various sources of information. Finally, because evidence in the decision process is assumed to accumulate over time, the time elapsing between the availability of the relevant and irrelevant information influences the magnitude of the Stroop effect.

\section{Parallel Distributed Processing Models}

The properties of automaticity as continuous, rather than all-or-none, and subject to attentional control are incorporated into a parallel distributed processing (PDP) model of the Stroop effect developed by Cohen, Dunbar, and McClelland (1990; see also Cohen, ServanSchreiber, \& McClelland, 1992). For the color Stroop task, the model consists of two processing pathways, one for color information and the other for color-word information (see Figure 11). The pathways contain input units that represent each possible color and color word (the colors and words RED and GREEN in Figure 11), task-demand units for each task alternative (in this case, color naming and word reading), intermediate units corresponding to the identities of the color names and the color words, and output units for the response alternatives (the vocal responses "red" and "green").

To simulate a trial, the task demand unit for the designated task is activated, in turn activating the intermediate units in the appropriate pathway. This sets the resting levels of activation for those units at a higher level than those of their counterparts in the other pathway. One consequence of this higher resting activation is to make the units relatively more sensitive than those that are not so activated. The occurrence of a stimulus is represented by a pattern of activation at the input units. This activation propagates to the intermediate units and then

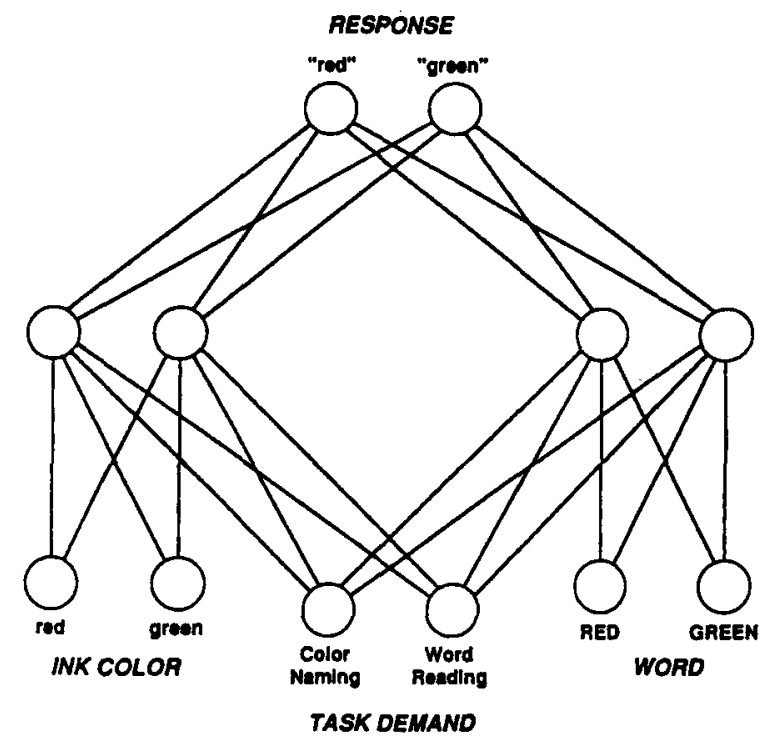

Figure 11. Cohen, Dunbar, and McClelland's (1990) parallel distributed processing model for the color Stroop effect. From "On the Control of Automatic Processes: A Parallel Distributed Processing Account of the Stroop Effect," by J. D. Cohen, K. Dunbar, \& J. L. McClelland, 1990, Psychological Review, 97, 332-361, Figure 1. Copyright 1990 by the American Psychological Association. Reprinted by permission.

to the output units. When the activation accumulating at one of the output units exceeds a threshold value, that response is made.

The network also has the capability to learn. It does so through application of the backpropagation learning algorithm (Rumelhart, Hinton, \& Williams, 1986), by which the connection strengths are adjusted to reduce the difference between the output pattern generated in response to an input and the output pattern that is desired. The model was trained initially by giving it practice at naming colors and reading color words, with the latter task receiving more practice than the former to reflect the fact that people have more experience with reading words than with naming colors.

Cohen et al. (1990) demonstrated that their model can account for many of the basic findings obtained with the Stroop task. These include that (1) interference is typically much greater than facilitation, (2) the Stroop effect is substantial for color naming but not for word reading, and (3) presenting the irrelevant color before the relevant word in the word-reading task by as much as $500 \mathrm{msec}$ still yields little or no Stroop effect. The model also addresses the relationship between learning and the time course of the processes involved in the Stroop task. Such learning produces decreases in RTs with practice, as well as changes in the interference patterns that occur as the relative processing speed of the two dimensions changes, as in MacLeod and Dunbar's (1988) study. Findings such as those of Logan and Zbrodoff $(1979,1982)$, which suggest that the Stroop effect can be altered by attentional strategies, can be accommodated within the model by alterations of the relative activations of the task-demand 
units. Two shortcomings of the model are that (1) although it fits mean RTs well, it does not capture changes in the RT distributions on which the means are based (Mewhort, Braun, \& Heathcote, 1992) and (2) no distinction is made between output modalities (O'Leary \& Barber, 1993). However, neither of these shortcomings seems insurmountable.

Phaf, Van der Heijden, and Hudson (1990) have also accounted for many of the same Stroop phenomena as have Cohen et al. (1990) with a similar PDP model. One distinction between the two is that, in Phaf et al.'s model, the Stroop asymmetry (that an irrelevant color word influences color naming but an irrelevant physical color does not affect word reading) results from an architectural property, rather than from differential practice. That is, in Phaf et al.'s model, the visual representation for stimulus color can activate a response only through a mediating semantic code (much like the intermediate units in Cohen et al.'s model). In contrast, there is a "privileged," direct connection between the visual representation of a word and its motor program that bypasses the semantic code. Thus, the Stroop asymmetry is produced by an asymmetry in the connections of the two stimulus dimensions to the output units. This model variation seems to capture better than does Cohen et al.'s the fact that there are privileged relations between specific stimulus modalities or codes and particular response modalities, but it has no learning mechanism to address changes that occur with practice. The most important point, though, is that models of the type proposed by Cohen et al. and Phaf et al. have considerable potential for characterizing the influence of irrelevant information, both location and nonlocation, on responding to relevant information.

\section{Summary}

The spatial Stroop effect can be obtained with integrated and nonintegrated stimuli for which both the relevant and the irrelevant dimensions signify spatial information. Most explanations ascribe the effect to the processing stage of response selection, with the interference that occurs when the relevant and irrelevant dimensions are incongruent typically attributed to response competition. The spatial Stroop effect is asymmetric, with the direction of the asymmetry being a function of the response modality: Stimulus location has a substantial effect on keypress responses to word meaning, but word meaning has only a slight effect on keypress responses to location; word meaning has a substantial effect on vocal responses to location, but location has only a slight effect on vocal responses to word meaning.

Evidence suggests that relative timing of the relevant and irrelevant information is important for the both the color and the spatial Stroop effects. For task variations that typically yield a reliable effect, the magnitude of the effect decreases when the irrelevant information is preexposed at increasing SOAs. For the task variations that do not typically yield a reliable effect, the effect is increased in magnitude somewhat by preexposing the ir- relevant dimension at intermediate SOAs of a few hundred milliseconds. The findings that (1) the response modality determines which stimulus dimension produces a significant Stroop effect and (2) presenting the irrelevant information before the relevant information has only a moderate influence on the magnitude of the Stroop effect for the conditions in which it is normally small suggest that the effect is not simply a function of a horse race between the two stimulus dimensions. These results are generally consistent with the translational model proposed by Virzi and Egeth (1985) in which verbal stimuli have privileged access to vocal responses and location stimuli have privileged access to keypress responses. However, the fact that conditions for which the irrelevant information should not be translated into response codes tend to show small amounts of Stroop interference suggests that translation is a matter of degree, rather than being all-or-none. That response activation may vary in degree is generally in agreement with studies of automaticity that similarly imply that automaticity be viewed as varying along a continuum.

The pattern of faster responses for congruent trials than for incongruent trials can be reversed by making the incongruent trials more frequent. Also, precuing whether the forthcoming stimulus will be congruent or incongruent facilitates responding. These and other results suggest that the direction and amount of Stroop interference is a function not only of the degree of automaticity of the preestablished $\mathrm{S}-\mathrm{R}$ associations but also of attentional strategies that involve the weightings of the relevant and irrelevant stimulus information in the response-selection process. The joint roles of automatic activation and attention are captured well by PDP models of the general type proposed by Cohen et al. (1990) and Phaf et al. (1990).

\section{A COMMON PROCESSING BASIS FOR THE SIMON AND SPATIAL STROOP EFFECTS}

Despite the fact that both the Simon and the spatial Stroop effects illustrate the influence of irrelevant location information on the processing of the relevant stimulus information, the two effects have been treated as different phenomena and investigated in distinct domains of studies. Moreover, as should be apparent from our literature review, research on the Simon effect has tended to emphasize different issues from that on the spatial Stroop effect. For the Simon effect, an assumption made by most researchers is that a spatial code for stimulus location is not always formed. Consequently, considerable effort has been devoted toward depicting the process by which the spatial code for the irrelevant location information is formed. In most cases, it has been assumed that if a spatial code is formed, it is guaranteed to show an effect on responding to the relevant stimulus dimension. In contrast, for the spatial Stroop effect, most researchers have presumed that the spatial code for the irrelevant location information is always formed and have focused on how interference arises 
from processing the spatial codes for the relevant and irrelevant stimulus dimensions.

In this section, we illustrate that - contrary to the way that they have typically been treated - the Simon and spatial Stroop effects are fundamentally the same. Because the taxonomy of S-R ensembles developed by Kornblum (1992, 1994) distinguishes the Simon task from the Stroop task, as well as from the Hedge and Marsh task, our arguments for the fundamental similarity of the Simon and spatial Stroop effects are based on consideration of this taxonomy.

\section{Kornblum's Taxonomy}

Kornblum's $(1992,1994)$ taxonomy is founded on the concept of dimensional overlap (i.e., similarity) and classifies $\mathrm{S}-\mathrm{R}$ ensembles into eight categories according to whether or not overlap exists between (1) the relevant and irrelevant stimulus dimensions, (2) the relevant stimulus dimension and the response dimension, and (3) the irrelevant stimulus dimension and the response dimension. According to Kornblum, overlap between the two stimulus dimensions (sometimes called $\mathrm{S}-\mathrm{S}$ congruity) is the critical factor distinguishing the Simon effect obtained with the original Simon task and its variations (e.g., the Hedge and Marsh paradigm) from the Stroop effect obtained with the spatial Stroop task.

In Kornblum's $(1992,1994)$ taxonomy, the Simon task is classified as a Type 3 ensemble, for which the relevant stimulus dimension has no overlap with the response dimension but the irrelevant stimulus dimension does. This is because the relevant stimulus dimension is nonspatial, whereas both the irrelevant stimulus dimension and the response dimension are spatial. In the information-processing model that Kornblum developed to accompany his taxonomy, stimulus location affects responding with Type 3 ensembles because a code for the corresponding response is automatically identified and programmed; RT is facilitated if this response code is verified as being the response indicated by the relevant stimulus dimension and inhibited if it is not.

The Hedge and Marsh (1975) task is classified as a Type 5 ensemble, rather than a Type 3 ensemble, because in addition to the irrelevant stimulus dimension overlapping with the response dimension (i.e., its location), the relevant stimulus dimension (color) overlaps with the relevant response dimension (color). From a response competition perspective, this overlap of the relevant dimensions would be a significant factor only if it influences the generation of response codes. Kornblum $(1992,1994)$ has not specified a processing model for the Type 5 ensembles, so how he would treat the overlap of the relevant $\mathrm{S}-\mathrm{R}$ dimensions is not evident. However, De Jong et al. (1994) have developed a processing model for the Hedge and Marsh task that, as described earlier, contains two components. The unconditional component reflects automatic activation of the response code for the location corresponding with that of the stimulus; the activation occurs shortly after presentation of the stimulus and then decreases. Due to its unconditional nature, this component would be expected to contribute similarly to the Simon effect in both the original Simon task and the Hedge and Marsh task. Consistent with this implication, the magnitude of the Simon effect varies in like manner as a function of relative processing speed in both tasks (De Jong et al., 1994; Hommel, 1993c; Lu \& Proctor, 1994).

The conditional component in De Jong et al.'s (1994) model, whose contribution does not vary as a function of time, is applicable only to the Hedge and Marsh task and is presumed to reflect generalization of the appropriate response-selection rule (identity or reversal) to the irrelevant location information. This component accounts for the reversal of the Simon effect with the incompatible, alternate-color mapping because application of the reversal rule produces a response code for the location opposite that of the stimulus. Whether it would contribute to the magnitude of the Simon effect with the compatible, same-color mapping in the Hedge and Marsh task is less clear. If subjects perform this version of the task by applying the identity rule, thus producing a response code for the location corresponding to that of the stimulus that is in addition to the code generated by the unconditional component, then the Simon effect should be enhanced. In fact, De Jong et al. found a larger Simon effect $(18 \mathrm{msec})$ for the same-color mapping of the Hedge and Marsh task than for a task in which the overlap of the relevant stimulus and response dimensions was eliminated by labeling the keys with " $x \times x x "$ and "++++" rather than with the colors $(6 \mathrm{msec})$. These results must be taken with caution, however, because (1) responses were slower on average in the modified task than in the Hedge and Marsh task, which could have resulted in less temporal overlap and hence a smaller effect and (2) the Simon effect in the Hedge and Marsh task was no larger than that obtained with the original Simon task in many studies. In sum, the same-color mapping of the Hedge and Marsh task may include a component not present in the Simon task that contributes to the magnitude of the Simon effect, but the effect follows a similar time course and can be attributed to response competition in both tasks.

The Stroop task is classified as a Type 8 ensemble in Kornblum's $(1992,1994)$ taxonomy. In addition to there being dimensional overlap between the irrelevant stimulus dimension and the response dimension and between the relevant stimulus dimension and the response dimension, there is also overlap between the relevant and irrelevant stimulus dimensions. For the version of the spatial Stroop task in which keypress responses are made to location words, dimensional overlap not only exists between the irrelevant stimulus location and response location and the relevant location word and response location but also between the relevant location word and irrelevant stimulus location. It is this overlap between the two stimulus dimensions that is emphasized in Kornblum's processing model as distinguishing the Stroop and Simon effects. In the model, the overlap of the relevant and irrelevant stimulus dimensions, which is present only in the Stroop task, produces interference at the stage of stimulus identification when the two dimensions are incongruent. 


\section{Is Overlap of the Relevant and Irrelevant Stimulus Dimensions Important?}

There is reason to think that the difference in overlap of the stimulus dimensions that differentiates the spatial Stroop task from the Simon task and the Hedge and Marsh task is not important. Hasbroucq and Guiard (1991) have argued that the Simon effect, both in the original Simon task and in the Hedge and Marsh version, is a spatial variant of the Stroop effect. According to them, the Simon effect arises from competition between the spatial codes produced by the relevant nonspatial stimulus dimension (e.g., color) and the irrelevant location dimension, although they attribute this competition to stimulus identification. If Hasbroucq and Guiard's conjecture about spatial codes being the source of the Simon and spatial Stroop effects is correct, then whether the relevant and irrelevant stimulus dimensions overlap is not of functional significance. Lu and Proctor (1994) obtained empirical confirmation of this conjecture by showing that the pattern of results for Simon and spatial Stroop tasks in the Hedge and Marsh paradigm are similar once effects of relative processing speed for the relevant stimulus dimension have been taken into account. More generally, our review of the literatures on the Simon and spatial Stroop effects in this paper has shown a striking similarity in the results obtained for each.

Kornblum (1994) reported evidence using Stroop-like and Simon-like stimuli that he interpreted as supporting the distinction between congruity effects arising in stimulus identification and in response selection. His experiment used stimuli that varied on three dimensions. Words were presented in white, centered in a rectangle that was divided into colored and dark halves. In all conditions, the rectangle color was the relevant stimulus dimension to which a keypress response was to be made. The words (color and neutral) and location of the colored half of the rectangle were varied both separately and together as irrelevant dimensions. Additionally, the irrelevant information either was presented simultaneous with the relevant information ( 0 -msec SOA) or preceded its onset by $200 \mathrm{msec}$. The effects of the two types of overlap, (1) color word with rectangle color and (2) relative location of the rectangle color with response location, were additive. Incongruity of the first type slowed responses more at the $200-\mathrm{msec}$ SOA than at the $0-\mathrm{msec}$ SOA, and incongruity of the second type showed the reversed relation. Kornblum interpreted these findings as support for the assumption of his model that congruity effects can arise in both stimulus identification and response selection.

There are two important points to note about Kornblum's (1994) study. First, the additivity demonstrated for effects of the overlap of color word with rectangle color and of rectangle location with response location does not in itself imply that the stage of stimulus identification is involved. That is, following additive-factors logic (Sternberg, 1969), this additivity suggests only that the two types of congruity influence different processing stages. To attribute the effect of overlap between color word and color to stimulus identification, it is necessary to show that this effect interacts with that of a marker variable that can be presumed to affect the stimulusidentification stage. Simon and Berbaum (1990) conducted an experiment similar to Kornblum's in which the variable of stimulus duration, which presumably would have its effect on the stimulus-identification stage, was also manipulated. Stimulus duration had additive effects with those of both color congruency and location correspondence, suggesting that neither effect can be attributed to stimulus identification. The second point is that, for the 0-msec SOA in Kornblum's study, responses were no slower when the color word and rectangle color were incongruent than when they were congruent. In other words, $\mathrm{S}-\mathrm{S}$ congruity, which is presumed by Kornblum to have its effect on stimulus identification, had no effect on RT under the standard condition for demonstrating the Stroop effect in which the irrelevant and relevant dimensions are presented simultaneously.

\section{Asymmetric Direction of Effects}

The Simon and spatial Stroop effects also are similar in that both show an asymmetry of the effects of one stimulus dimension, when irrelevant, on responding to the other. For the Simon effect, the irrelevant information of stimulus location affects the time to respond to the relevant symbolic attribute (e.g., color of stimulus). However, when stimulus location is the relevant dimension and the symbolic attribute is the irrelevant dimension, the irrelevant information does not influence the pattern of performance. For the spatial Stroop effect, the irrelevant information of stimulus location affects the time to respond manually to the relevant stimulus dimension of location word; however, when location word is irrelevant, it does not have much effect on manual responses to stimulus location. For vocal responses, this asymmetry is reversed.

Kornblum's $(1992,1994)$ taxonomy predicts the asymmetry for the Simon task as a consequence of the fact that dimensional overlap exists only between stimulus location and response location. An irrelevant dimension that has no overlap with response location should not affect responding, so the Simon effect will occur only when the irrelevant dimension is stimulus location. However, the taxonomy does not provide a basis for predicting the similar asymmetry that is obtained for the spatial Stroop task, because, in that task, both the relevant and the irrelevant stimulus dimensions overlap with the response dimension, regardless of which one is defined as irrelevant. The problem is that, although Kornblum assumed that dimensional overlap can vary in degree and that the difference between compatible and incompatible mappings of the stimulus and response elements is an increasing function of dimensional overlap, this assumption is not reflected in his taxonomy. Because the taxonomy treats dimensional overlap as all-or-none, it implies that the asymmetry should occur only when just one stimulus dimension overlaps with the response dimension, as in the Simon task. Assuming that dimen- 
sional overlap varies in degree, then the asymmetry for the spatial Stroop effect can be seen to have the same basis as that for the Simon effect. In both cases, the location dimension overlaps more with keypress responses than does the other stimulus dimension. Thus, the response activation produced by the irrelevant dimension will be greater when that dimension is location than when it is not.

\section{Summary}

On the whole, the evidence seems to indicate that the Simon and spatial Stroop effects are not fundamentally different and have a similar processing basis. The overlap between stimulus dimensions that is present in the Stroop task but not in the Simon task or the Hedge and Marsh task does not appear to be of functional significance, and the two effects exhibit many similarities, such as the asymmetries of direction. Because the crucial relation in all of the tasks is between the irrelevant stimulus location and the response, it seems reasonable to attribute both the Simon and the spatial Stroop effect to response selection.

\section{PROCESSING IRRELEVANT LOCATION INFORMATION}

Our review of the research literatures on the Simon and spatial Stroop effects identified several basic factors involved in the processing of irrelevant location information. These factors, which provide insight into the direction that processing models of these effects must take, include (1) dependence on response modality, (2) spatial coding, (3) relative timing, and (4) allocation of attention.

\section{Dependence on Response Modality}

Virtually all studies of the Simon effect have used keypress responses or other response sets with physical spatial characteristics. As a consequence, a person reading the literature on the Simon effect would get the impression that response modality is of little importance. However, studies of the spatial Stroop effect have shown quite convincingly that the asymmetry evident with keypress responses (i.e., a substantial Stroop effect when location is irrelevant and the symbolic information relevant, but not when these relations are the opposite) reverses for vocal "left"-"right" responses (O'Leary \& Barber, 1993; Shimamura, 1987; Virzi \& Egeth, 1975). With vocal responses, the Stroop effect is evident primarily when the symbolic information is irrelevant and the location is relevant.

This reversal of the asymmetry when response modality changes is consistent with Virzi and Egeth's (1985) translational model in which there are two processing systems, one for verbal stimuli and vocal responses and the other for location stimuli and keypress responses. In terms of the model, the explanation for the asymmetry and its dependence on response modality is that a Stroop effect will occur only when the irrelevant stimulus information is automatically processed to the response- selection stage within the same system and the relevant stimulus information must be translated into that system. Because in Virzi and Egeth's model translation of the irrelevant stimulus information across processing systems is not supposed to occur, there should be no effect of irrelevant information when the response is in a different system.

However, several studies have shown this implication of Virzi and Egeth's (1985) model to be incorrect. The model seemingly predicts that no Stroop effect will occur when keypress responses are made to either color word or color in the color Stroop task because neither of these dimensions is in the same system as the keypress response. Yet, such effects are customarily obtained (e.g., Keele, 1972; Melara \& Mounts, 1993). Moreover, even in the spatial Stroop task, the evidence suggests that irrelevant information is not blocked out entirely from response selection in situations for which translation of that information across systems would be required. For example, a significant effect of irrelevant location, although small in magnitude, has been found even when vocal responses were made to location words (O'Leary \& Barber, 1993). Similarly, location words have been found to affect keypress responses to arrow stimuli significantly when the word precedes the arrow by a few hundred milliseconds (Lu \& Proctor, 1995). Findings such as these suggest that rather than translation being all-or-none, as implied by Virzi and Egeth's model, activation of responses by irrelevant information is one of degree. The notion that irrelevant information can activate responses to varying extents is in agreement with the proposal that dimensional overlap varies along a continuum (Kornblum et al., 1990) and evidence that automaticity is not all-or-none (MacLeod \& Dunbar, 1988). The results regarding response modality thus implicate preexisting associations of stimulus attributes to various response modalities that differ in strength.

\section{Spatial Coding}

Although keypress responses to relevant stimulus information are typically affected by an irrelevant location attribute, one contribution of research on the Simon effect is to show that such effects occur only if stimulus location is coded. Wallace (1971) provided evidence that the Simon effect arises from spatial codes generated by the irrelevant stimulus location information and in response to the relevant symbolic stimulus information. Reactions will be faster when the two spatial codes correspond and slower when they do not. This general view that the Simon effect arises from spatial coding of the irrelevant information of stimulus location has been accepted by most researchers, and considerable effort has been devoted to determining when and how this coding occurs. The evidence has indicated quite clearly that the frame of reference for spatial coding is not fixed with respect to the performer (e.g., Umiltà \& Liotti, 1987; Umiltà \& Nicoletti, 1985). In many situations spatial coding of the stimulus set is based on the relative locations in which a stimulus may occur. Likewise, spatial 
coding of the response set is a function of the relative locations of the alternative responses. However, spatial coding may also occur in terms of egocentric hemispace (Umiltà \& Liotti, 1987), with respect to the location of a marker in a complex array (e.g., Nicoletti \& Umiltà, 1989), or as a function of configurational cues in the display (e.g., Hommel, in press). There is some evidence indicating that multiple spatial codes, all of which contribute to the Simon effect, may be produced by a single stimulus (De Jong et al., 1994; Lamberts et al., 1991).

The basis for spatial coding is currently a topic of heated debate. One possibility is that the spatial codes are generated when there is a lateral shift of attention to the location occupied by the stimulus (Stoffer, 1991; Umiltà \& Nicoletti, 1992). Referential coding, in which comparison of the target stimulus to a reference frame or object results in the spatial coding of the stimulus location, provides another possible account for the generation of spatial codes (Hommel, 1993d; Umiltà \& Nicoletti, 1985 ). Neither the attention shifting nor the referential coding account, as presently developed, seems able to handle the full range of data; for example, effects of irrelevant location can be substantial for situations in which a lateral shift of attention should not occur (Weeks et al., $1995)$ and smaller when an explicit referent object (a fixation point) is present than when one is not (Proctor $\& \mathrm{Lu}, 1994)$. It is likely that several mechanisms, some obligatory and some not, contribute to spatial coding.

\section{Relative Timing of the Relevant and Irrelevant Information}

For both the Simon and the spatial Stroop effect, the relative timing of the relevant and irrelevant stimulus information at response selection has been hypothesized to be a critical factor in determining the occurrence of the effects. Horse race accounts for the spatial Stroop effect claim that the irrelevant dimension will influence performance when it is processed faster than the relevant dimension, because the faster dimension enters the singlechannel response buffer first. If order of entry into a response buffer were the crucial factor, it should be possible to produce a substantial Stroop effect in conditions that do not normally yield one by presenting the irrelevant information prior to the relevant information. This prediction has not been confirmed for vocal responses to location words or keypress responses to locations for the spatial Stroop task (Virzi \& Egeth, 1985), or for the analogous conditions in the color Stroop task (Glaser \& Glaser, 1982), although Stroop effects of relatively small magnitude do occur at intervals in the range of $200-300 \mathrm{msec}$ (Glaser \& Glaser, 1982; Lu \& Proctor, 1995).

For situations that typically produce a substantial Stroop or Simon effect, the magnitude of the effect should either be enhanced or remain constant when the irrelevant information is precued, if priority of entry into a response buffer is the sole determinant of interference. For the Stroop color-naming effect, the typical pattern is that the effect is reduced but not completely eliminated at long SOAs (Dyer, 1971; Glaser \& Glaser, 1982). We found no studies for the spatial Stroop effect in which precuing was used with tasks that required vocal responses to locations. However, for a task that required keypress responses to location words, $\mathrm{Lu}$ and Proctor (1995) showed that an irrelevant arrow pointing left or right produced a 25-msec Simon effect with simultaneous presentation and that this effect decreased to approximately $15 \mathrm{msec}$ at SOAs of 300-500 msec. Similarly, Goolkasian (1989) obtained a Stroop effect of $20 \mathrm{msec}$ from distractor location words presented simultaneously with the left or right movement of a target cursor to which a left or right keypress was to be made and found this effect to be reduced in magnitude when the onset of the location word preceded the target movement. Finally, for the Simon effect, a precue indicating the likely stimulus location in advance of the onset of the stimulus does not increase the magnitude but either reduces it or leaves it unaltered (Hommel, 1993a; Proctor et al., 1992; Stoffer \& Yakin, 1994; Verfaellie et al., 1988). Thus, the evidence is inconsistent with the proposal that the Simon or spatial Stroop effect is determined solely by prior entry of the response associated with the irrelevant information into a response buffer.

Hommel's (1993c) temporal overlap hypothesis is based on the concept of relative timing but assumes that it is the overlap of response activation functions for the relevant and irrelevant information that determines the magnitude of the effect produced by the irrelevant information. Because activation for the irrelevant information first increases and then decreases, this hypothesis predicts that the influence of the irrelevant information will be reduced or eliminated at long SOAs for conditions that typically produce a substantial effect, as most studies show. Although not directly predicted by the account, the temporal overlap hypothesis also can accommodate the finding that precuing irrelevant information in situations for which a Simon or Stroop effect is typically not evident produces an effect of only relatively small magnitude. The necessary assumption to explain this result is that the activation produced by the irrelevant information in such cases is sufficiently small, relative to that produced by the relevant information, that it has comparatively little impact on performance even when the temporal overlap is maximal.

One implication of any relative timing account, including the temporal overlap hypothesis, is that altering the relative difficulty of processing the relevant and irrelevant information when presented simultaneously should produce results similar to those obtained by precuing. This implication has been confirmed in several studies. Hommel (1993c) found that manipulations that slowed the processing of the relevant dimension reduced the effect of the irrelevant location dimension in the Simon task. Similarly, Lu and Proctor (1994) showed that when keypress responses were made to sets of color or location-word stimuli, the effect of irrelevant location was a decreasing function of the overall mean RT, and De Jong et al. (1994) showed that within the distributions of RTs from a single experimental condition, the 
Simon effect was a decreasing function of RT. In the Stroop color-naming task, Dunbar and MacLeod (1984) demonstrated that slowing reading of the irrelevant color words by degrading the display did not eliminate the Stroop effect but did tend to reduce it. This pattern is comparable to that observed when SOA is varied in Stroop color-naming tasks. Dunbar and MacLeod also found that degrading the display did not introduce an effect of irrelevant colors on word reading. This outcome also is in agreement with the finding that precuing an irrelevant color has only a small effect on reading a relevant color word. In summary, both the indirect and direct manipulations of timing seem to indicate that relative timing is important.

One of the more intriguing recent findings is that the time course of the reversed Simon effect obtained with the alternate-color mapping in the Hedge and Marsh task is opposite that of the standard Simon effect (De Jong et al., 1994; Lu \& Proctor, 1994). Whereas the standard Simon effect decreases with delays in responding, the reversed Simon effect increases. At present, De Jong et al.'s two-process account, in which the effects are produced by the sum of two processes-an unconditional one that decreases with time and a conditional one that does not-provides the best explanation of the opposing time courses.

\section{Role of Attention}

In the typical Stroop or Simon task, congruent and incongruent trials occur equally often and randomly. Logan and Zbrodoff $(1979,1982)$ showed that the usual pattern of faster responses on congruent trials than on incongruent trials is affected by manipulations that should influence attentional strategies. Specifically, the benefit for congruent trials is increased by making them relatively more frequent than incongruent trials or by providing a precue that designates the forthcoming stimulus as congruent. More strikingly, the benefit is reversed by making the incongruent trials much more frequent than the congruent trials or by precuing them. When the likely trial type is known in advance due to either precuing or extreme differences in relative frequency, the stimulus dimension that is nominally irrelevant becomes informative because its relation to the correct response is known. It therefore makes sense that subjects adopt strategies that make use of this information.

Logan and Zbrodoff $(1979,1982)$ proposed that the effects of relative frequency and precuing could reflect how subjects divide attention between the relevant and irrelevant dimensions. Specifically, they suggested that the magnitude of attentional weighting devoted to the "irrelevant" dimension would increase as the incongruent trials became relatively more likely than the congruent trials, or vice versa, because of this dimension's predictive validity. The strategy of attending to the irrelevant dimension when its likely relation to the relevant information is known seems to be effective only for task variations in which the irrelevant dimension would typically influence responding: With the keypress-response-to- position task, relative frequency did not have the same type of effect as it did on the keypress-response-to-word task (Logan \& Zbrodoff, 1979). Moreover, more than just an attentional weighting is likely involved in the strategies adopted, because congruent trials show a greater benefit of being primed or made more frequent than do incongruent trials. This finding suggests an additional strategic factor of the type proposed by Van Duren and Sanders (1988) and Stoffels (1995): Automatically activated, natural response tendencies must be inhibited unless they will likely be correct. In the case of the spatial Stroop task, the initial tendency to respond at the location coinciding with that of the stimulus is known to likely be correct only when corresponding trials predominate or are precued, thus affording an extra advantage for those situations.

\section{CONCLUSION}

None of the accounts developed for the Simon effect or the spatial Stroop effect seems capable of handling the majority of findings from both task domains without significant modification. However, many of the central features of these accounts are supported by the existing evidence, and a model that combines several of these features in a principled manner would seem to be most promising. The major factors reviewed in the previous section set limits on the direction that models of the Simon and spatial Stroop effects can take.

The fact that, for keypress responses, irrelevant stimulus location has a much larger effect on responding to a location word than does an irrelevant location word on responding to stimulus location, with the reversed pattern occurring for vocal responses, indicates that models of the effects cannot be based on stimulus properties alone. Instead, the representations of verbal and nonverbal stimuli, as well as vocal and manual responses, should be construed as being organized into distinct systems, as in Virzi and Egeth's (1985) model. However, the data now indicate relatively unambiguously that even for the conditions in which large effects are not evident-that is, responding vocally to location words in the presence of a nonverbal location dimension or manually to stimulus location in the presence of an irrelevant location wordthe irrelevant information has some effect on performance. The implication of these findings is that the verbal and nonverbal stimulus systems are connected directly to both the manual and the vocal response system but to different extents. This is in general agreement with network models of the type proposed by Cohen et al. (1990) and Phaf et al. (1990), as well as suggestions of O'Leary and Barber (1993).

More specifically, stimulus attributes are more strongly associated with their corresponding responses in the "compatible" response modality than in the "incompatible" modality. Consequently, irrelevant information will activate responses in different response systems to different degrees, and the strength of the activation produced by the irrelevant information will set the upper 
limit for the magnitude of effect that this information can have on responses to the relevant information. If the response activation produced by the relevant dimension is much greater than that produced by the irrelevant dimension, as in the case of the relation between irrelevant verbal location information and keypress responses, then the maximum effect that this irrelevant information can have on performance will be relatively small. However, if the response activation produced by the irrelevant information is relatively strong, as in the case of the relation between irrelevant verbal location information and vocal responses, then its effect may be substantial.

Models must take into account not only the strength of response activation produced by the irrelevant information but also the dynamics of the timing of this activation with respect to that produced by the relevant information. Many findings now suggest that the response activation produced by the irrelevant information decreases, due to either intentional inhibition or passive decay, after it reaches its maximum. The maximal activation of the response code occurs quickly when the irrelevant information is highly compatible with the response modality, as in the case of stimulus locations and keypresses, relative to when it is not, as in the case of location words and keypresses. An account based on just the principles of differences in association strength and relative timing seems adequate to handle many of the findings obtained for the Simon and spatial Stroop tasks. It may be necessary, however, to add a nontemporal component, as in De Jong et al.'s (1994) two-process model, to accommodate the pattern of increasing effects with slower responding that occurs in the Hedge and Marsh (1975) task for situations in which the reversal rule applies.

Any complete model for the effect of irrelevant location information on performance must address the conditions under which a spatial code will be produced. Although the process (or processes) that produces spatial codes is still a point of contention, the codes often are a function of relative location. There is some evidence to suggest that models must allow for multiple spatial codes to be produced for a single stimulus event (e.g., De Jong et al., 1994; Lamberts et al., 1992), but this evidence is not yet conclusive.

Perhaps the most neglected aspect that any comprehensive model will have to address is the role of strategies. People seem quite adept at modifying their strategies as task situations and demands change, even to the extent of showing an advantage in some situations for the trials that are typically the most difficult-that is, those on which the information defined as irrelevant is incongruent with that defined as relevant. The strategic component involves in part varying the attentional weightings given to the different sources of information. This component can be instantiated readily within a network model of the type developed by Cohen et al. (1990) for the color Stroop task in which effects of task instructions are represented by color-naming and word-reading task-demand units that can be activated to varying degrees to produce attentional effects.
The view that attention can be represented by temporary changes in sensitivity within the preexisting network implies that the effects of irrelevant location information should be similar to those obtained when location is relevant, only reduced in magnitude. Consistent with this view, correspondence of stimulus and response locations produces a similar pattern of effects regardless of whether stimulus location is relevant or irrelevant, with the magnitude of these effects usually less when location is irrelevant (i.e., in the Simon task) than when it is relevant (i.e., in a spatial compatibility task; Umiltà and Nicoletti, 1990).

In summary, it is clear that many factors go into determining the magnitude of effect that irrelevant location information, verbal or nonverbal, has on performance. Any comprehensive model for the influence of irrelevant location information on performance will have to characterize the dynamic interactions of these factors. At present, network models of the type that have been applied with some success to the Stroop color-naming task seem most suited to doing so.

\section{REFERENCES}

AREND, U., \& WANDMACHER, J. (1987). On the generality of logical recoding in spatial interference tasks. Acta Psychologica, 65, 193-210.

Barber, P. J., O'Leary, M. J., \& Simon, J. R. (1994). Defining stimulus congruity. A rejoinder to Guiard, Hasbroucq, and Possamai (1994). Psychological Research/Psychologische Forschung, 56, 213-215.

BASHINSKI, H. S., \& BaChaRACH, V. R. (1980). Enhancement of perceptual sensitivity as the result of selectively attending to spatial locations. Perception \& Psychophysics, 28, 241-248.

BREBNER, J. (1979). The compatibility of spatial and non-spatial relationships. Acta Psychologica, 43, 23-32.

Clark, H. H., \& Brownell, H. H. (1975). Judging up and down. Journal of Experimental Psychology: Human Perception \& Performance, 1, 339-352.

Cohen, J. D., Dunbar, K., \& McClelland, J. L. (1990). On the control of automatic processes: A parallel distributed processing account of the Stroop effect. Psychological Review, 97, 332-361.

Cohen, J. D., Servan-Schreiber, D., \& McClelland, J. L. (1992). A parallel distributed processing approach to automaticity. American Journal of Psychology, 105, 239-269.

Crafr, J. L., \& Simon, J. R. (1970). Processing symbolic information from a visual display: Interference from an irrelevant directional cue. Journal of Experimental Psychology, 83, 415-420.

DALRYMPLE-ALFORD, E. C. (1968). Interlingual interference in a colornaming task. Psychonomic Science, 10, 215-216.

DALRYMPLE-ALFORD, E. C. (1972). Associative facilitation and interference in the Stroop color-word task. Perception \& Psychophysics, 11, 274-276.

Dalrymple-Alford, E. C., \& AzKoul, J. (1972). The locus of interference in the Stroop and related tasks. Perception \& Psychophysics, 11, 385-388.

De Jong, R., Liang, C.-C., \& Lauber, E. (1994). Conditional and unconditional automaticity: A dual-process model of effects of spatial stimulus-response correspondence. Journal of Experimental Psychology: Human Perception \& Performance, 20, 731-750.

Dunbar, K. N., \& MACLEOD, C. M. (1984). A horse race of a different color: Stroop interference patterns with transformed words. Journal of Experimental Psychology: Human Perception \& Performance, 10, 622-639.

DYER, F. N. (1971). The duration of word meaning responses: Stroop interference for different preexposures of the word. Psychonomic Science, 25, 229-231.

DYER, F. N. (1972). Latencies for movement naming with congruent 
and incongruent word stimuli. Perception \& Psychophysics, 11, 377 . 380 .

DYER, F. N. (1973). The Stroop phenomenon and its use in the study of perceptual, cognitive, and response processes. Memory \& Cognition, $1,106-120$.

DYER, F. N. (1974). Stroop interference with long preexposures of the word: Comparison of pure and mixed preexposure sequences. Bulletin of the Psychonomic Society, 3, 8-10.

ERIKSEN, C. W., \& HofFMAN, J. E. (1972). Some characteristics of selective attention in visual perception determined by vocal reaction time. Perception \& Psychophysics, 11, 169-171.

Flowers, J. H., \& STOUP, C. M. (1977). Selective attention between words, shapes and colors in speeded classification and vocalization tasks. Memory \& Cognition, 5, 299-307.

Fox, L. A., Shor, R. E., \& Steinman, R. J. (1971). Semantic gradients and interference in naming color, spatial direction, and numerosity. Journal of Experimental Psychology, 91, 59-65.

GlaSER, M. O., \& DolT, M. O. (1977). A functional model to localize the conflict underlying the Stroop phenomenon. Psychological Research/Psychologische Forschung, 39, 287-310.

GLASER, M. O., \& GLASER, W. R. (1982). Time course analysis of the Stroop phenomenon. Journal of Experimental Psychology: Human Perception and Performance, 8, 875-894.

Glaser, W. R., \& Glaser, M. O. (1989). Context effects in Stroop-like word and picture processing. Journal of Experimental Psychology: General, 118, 13-42.

GoolKasIAN, P. (1989). Target and distractor processing at several retinal locations. Bulletin of the Psychonomic Society, 27, 231-233.

GreENWALD, A. G. (1972). On doing two things at once: Time sharing as a function of ideomotor compatibility. Journal of Experimental Psychology, 94, 52-57.

Grice, G. R., CANHAM, L., \& Boroughs, J. M. (1984). Combination rule for redundant information in reaction time tasks with divided attention. Perception \& Psychophysics, 35, 451-463.

Grice, G. R., Canham, L., \& GWYNne, J. W. (1984). Absence of a redundant-signals effect in a reaction time task with divided attention. Perception \& Psychophysics, 36, 565-570.

GuIARD, Y. (1983). The lateral coding of rotations: A study of the Simon effect with wheel-rotation responses. Journal of Motor Behavior, $15,331-342$.

Guiard, Y., Hasbrouce, T., \& Possamai, C.-A. (1994). Stimulus congruity, irrelevant spatial SR correspondence, and display-control arrangement correspondence. Psychological Research/Psychologische Forschung, 56, 210-212.

HARVEY, N. (1984). The Stroop effect: Failure to focus attention or failure to maintain focusing? Quarterly Journal of Experimental Psychology, 36, 89-115.

HASBRoUCQ, T., \& GUIARD, Y. (1991). Stimulus-response compatibility and the Simon effect: Toward a conceptual clarification. Journal of Experimental Psychology: Human Perception \& Performance, $17,246-266$.

HasbroucQ, T., Guiard, Y., \& Kornblum, S. (1989). The additivity of stimulus-response compatibility with the effects of sensory and motor factors in a tactile choice reaction time task. Acta Psychologica, 72, 139-144.

Hasbrouce, T., \& PossamaI, C.-A. (1994). What can a precue enhance? An analysis of the experiments of Proctor, $\mathrm{Lu}$, and Van Zandt (1992). Acta Psychologica, 85, 235-244.

HEDGE, A., \& MARSH, N. W. A. (1975). The effect of irrelevant spatial correspondences on two-choice response-time. Acta Psychologica, 39, 427-439.

Hock, H. S., \& EgETh, H. (1970). Verbal interference with encoding in a perceptual classification task. Journal of Experimental Psychology, 83, 299-303.

HofFMAN, J. E., \& Nelson, B. (1981). Spatial selectivity in visual search. Perception \& Psychophysics, 30, 283-290.

HoMmeL, B. (1993a, November). The effects of spatial cues on visual attention, response selection, and spatial compatibility. Poster presented at the 34th annual meeting of the Psychonomic Society, Washington, DC.

HOMMEL, B. (1993b). Inverting the Simon effect by intention: Determinants of direction and extent of effects of irrelevant spatial infor- mation. Psychological Research/Psychologische Forschung, 55, 270-279.

HOMMEL, B. (1993c). The relationship between stimulus processing and response selection in the Simon task: Evidence for a temporal overlap. Psychological Research/Psychologische Forschung, 55, 280-290.

Hommel, B. (1993d). The role of attention for the Simon effect. Psychological Research/Psychologische Forschung, 55, 208-222.

HoMmeL, B. (1994a). Effects of irrelevant spatial S-R compatibility depend on stimulus complexity. Psychological Research/Psychologische Forschung, 56, 185-195.

HOMMEL, B. (1994b). Spontaneous decay of response-code activation. Psychological Research/Psychologische Forschung, 56, 261-268.

HOMMEL, B. (in press). Stimulus-response compatibility and the Simon effect: Toward an empirical clarification. Journal of Experimental Psychology: Human Perception \& Performance.

JAMES, W. (1950). The principles of psychology (Vol. 2). New York: Dover. (Original work published 1890)

JONIDES, J., \& MACK, R. (1984). On the cost and benefit of cost and benefit. Psychological Bulletin, 96, 29-44.

KEELE, S. (1972). Attention demands of memory retrieval. Journal of Experimental Psychology, 93, 245-248.

KLEIN, G. S. (1964). Semantic power measured through the interference of words with color-naming. American Journal of Psychology, 77, 576-588.

KoRNBLUM, S. (1992). Dimensional overlap and dimensional relevance in stimulus-response and stimulus-stimulus compatibility. In G. Stelmach \& J. Requin (Eds.), Tutorials in motor behavior II (pp. 743777). Amsterdam: North-Holland.

KoRnBLUM, S. (1994). The way irrelevant dimensions are processed depends on what they overlap with: The case of Stroop-and Simonlike stimuli. Psychological Research/Psychologische Forschung, 56, 130-135.

Kornblum, S., HasbroucQ, T., \& Osman, A. (1990). Dimensional overlap: Cognitive basis for stimulus-response compatibility-A model and taxonomy. Psychological Review, 97, 253-270.

Lamberts, K., TAVernier, G., \& D'YDEwalle, G. (1992). Effect of multiple reference points in spatial stimulus-response compatibility. Acta Psychologica, 79, 115-130.

LOGAN, G. D. (1980). Attention and automaticity in Stroop and priming tasks: Theory and data. Cognitive Psychology, 12, 523-553.

LOGAN, G. D. (1994). Spatial attention and the apprehension of spatial relations. Journal of Experimental Psychology: Human Perception \& Performance, 20, 1015-1036.

LOGAN, G. D., \& ZBRODOFF, N. J. (1979). When it helps to be misled: Facilitative effects of increasing the frequency of conflicting stimuli in a Stroop-like task. Memory \& Cognition, 7, 166-174.

LOGAN, G. D., \& ZBRODOFF, N. J. (1982). Constraints on strategy construction in a speeded discrimination task. Journal of Experimental Psychology: Human Perception \& Performance, 8, 502-520.

LogAN, G. D., ZBRODOFF, N. J., \& FosteY, A. R. W. (1983). Costs and benefits of strategy construction in a speeded discrimination task. Memory \& Cognition, 11, 485-493.

LU, C.-H., \& PROCTOR, R. W. (1994). Processing of an irrelevant location dimension as a function of the relevant stimulus dimension. Journal of Experimental Psychology: Human Perception \& Performance, 20, 286-298.

LU, C.-H., \& ProctoR, R. W. (1995). Effects of $S-R$ association strength and relative timing on the processing of relevant and irrelevant information. Manuscript submitted for publication.

MACLEOD, C. M. (1991). Half a century of research on the Stroop effect: An integrative review. Psychological Bulletin, 109(2), 163-203.

MacLeod, C. M., \& Dunbar, K. (1988). Training and Stroop-like interference: Evidence for a continuum of automaticity. Journal of Experimental Psychology: Learning, Memory, \& Cognition, 14, 126135.

MCCANN, R. S., \& Johnston, J. C. (1992). Locus of the single-channel bottleneck in dual-task interference. Journal of Experimental Psychology: Human Perception \& Performance, 18, 471-484.

McClaIN, L. (1983). Stimulus-response compatibility affects auditory Stroop interference. Perception \& Psychophysics, 33, 266-270.

Melara, R. D., \& Mounts, J. R. W. (1993). Selective attention to 
Stroop dimensions: Effects of baseline discriminability, response mode, and practice. Memory \& Cognition, 21, 627-645.

Mewaldt, S. P., Connelly, C. L., \& Simon, J. R. (1980). Response selection in choice reaction time: Test of a buffer model. Memory \& Cognition, 8, 606-611.

Mewhort, D. J. K., Braun, J. G., \& HeathCote, A. (1992). Response time distributions and the Stroop task: A test of the Cohen, Dunbar, and McClelland (1990) model. Journal of Experimental Psychology: Human Perception \& Performance, 18, 872-882.

MORTON, J., \& CHAMBERS, S. M. (1973). Selective attention to words and colours. Quarterly Journal of Experimental Psychology, 25, 387-397.

Nicoletti, R., Anzola, G. P., Luppino, G., Rizzolatti, G., \& UMILTÀ, C. (1982). Spatial compatibility effects on the same side of the body midline. Journal of Experimental Psychology: Human Perception \& Performance, 8, 664-673.

Nicoletti, R., \& Umiltà, C. (1989). Splitting visual space with attention. Journal of Experimental Psychology: Human Perception \& Performance, 15, 164-169.

Nicoletti, R., \& Umiltà, C. (1994). Attention shifts produce spatial stimulus codes. Psychological Research/Psychologische Forschung, 56, 144-150.

NisSEN, M. J. (1985). Accessing features and objects: Is location special? In M. I. Posner \& D. S. Marin (Eds.), Attention and performance XI (pp. 205-219). Hillsdale, NJ: Erlbaum.

O'LeARY, M. J., \& Barber, P. J. (1993). Interference effects in the Stroop and Simon paradigms. Journal of Experimental Psychology. Human Perception \& Performance, 19, 830-844.

O'LEARY, M. J., \& BARBER, P. J. (1994). Stimulus congruence and the Simon effect. Psychological Research/Psychologische Forschung, 56, 196-202.

O'Leary, M. J., Barber, P. J., \& Simon, J. R. (1994). Does stimulus correspondence account for the Simon effect? Comments on Has broucq and Guiard (1991). Psychological Research/Psychologische Forschung, 56, 203-209.

PALEF, S. R. (1978). Judging pictorial and linguistic aspects of space. Memory \& Cognition, 6, 70-75.

PALEF, S. R., \& Olson, D. R. (1975). Spatial and verbal rivalry in a Stroop-like task. Canadian Journal of Psychology, 29, 201-209.

Phaf, R. H., VAN der Heuden, A. H. C., \& Hudson, P. T. W. (1990). SLAM: A connectionist model for attention in visual selection tasks. Cognitive Psychology, 22, 273- 341 .

POSNER, M. I. (1978). Chronometric explorations of mind. Hillsdale, NJ: Erlbaum.

PosNer, M. I., \& SNYDER, C. R. R. (1975). Attention and cognitive control. In R. L. Solso (Ed.), Information processing and cognition: The Loyola symposium (pp. 55-85). Hillsdale, NJ: Erlbaum.

PosNer, M. I., SNYDER, C. R. R., \& Davidson, B. J. (1980). Attention and the detection of signals. Journal of Experimental Psychology: General, 109, 160-174.

Prinz, W., Aschersleben, G., Hommel, B., \& Vogt, S. (1993). Handlugen als Ereignese. In D. Dörner \& E. van der Meer (Eds.), Gedächtnis. Trends, probleme, perspektiven. Heidelberg: Springer-Verlag.

PROCTOR, R. W. (1978). Sources of color-word interference in the Stroop color-naming task. Perception \& Psychophysics, 23, 413-419.

PROCTOR, R. W. (1981). A unified theory for matching task phenomena. Psychological Review, 88, 291-326.

Proctor, R. W., \& DutTA, A. (1993). Do the same stimulus-response relations influence choice reactions initially and after practice? Journal of Experimental Psychology: Learning, Memory, \& Cognition, $19,922-930$.

ProctoR, R. W., \& LU, C.-H. (1994). Referential coding and attention shifting accounts of the Simon effect. Psychological Research/Psychologische Forschung, 56, 185-195.

Proctor, R. W., LU, C.-H., \& VAN ZANDT, T. (1992). Enhancement of the Simon effect by response precuing. Acta Psychologica, 74, 53-74

Proctor, R. W., Lu, C.-H., Wang, H., \& DutTa, A. (in press). Activation of response codes to varying degrees by relevant and irrelevant information. Acta Psychologica.

PROCTOR, R. W., \& REEVE, T. G. (Eds.) (1990). Stimulus-response compatibility: An integrated perspective. Amsterdam: North-Holland. RIGGIO, L., GaWRYSZEWSKI, L. G., \& UMILTÀ, C. (1986). What is crossed in crossed-hand effects? Acta Psychologica, 62, 89-100.
Rumelhart, D. E., Hinton, G. E., \& McClelland, J. L. (1986). A general framework for parallel distributed processing. In D. E. Rumelhart, J. L. McClelland, \& PDP Research Group (Eds.), Parallel distributed processing: Explorations in the microstructure of cognition (Vol. 1, pp. 318-362). Cambridge, MA: MIT Press.

SCHNEIDER, W., \& Fisk, A. D. (1982). Degree of consistent training: Improvements in search performance and automatic process development. Perception \& Psychophysics, 31, 160-168.

Seymour, P. H. (1973). Stroop interference in naming and verifying spatial locations. Perception \& Psychophysics, 14, 95-100.

SHIFFrIN, R. M., \& SCHNEIDER, W. (1977). Controlled and automatic human information processing: II. Perceptual learning, automatic attending and a general theory. Psychological Review, 84, 127-190.

Shimamura, A. P. (1987). Word comprehension and naming: An analysis of English and Japanese orthographies. American Journal of Psychology, 100, 15-40.

SHOR, R. E. (1970). The processing of conceptual information on spatial directions from pictorial and linguistic symbols. Acta Psychologica, 32, 346-365.

ShOR, R. E., Hatch, R. P., Hudson, L. J., Landrigan, D. T., \& ShafFER, H. J. (1972). Effect of practice on a Stroop-like spatial directions task. Journal of Experimental Psychology, 94, 168-172.

SimON, J. R. (1990). The effects of an irrelevant directional cue on human information processing. In R. W. Proctor \& T. G. Reeve (Eds.), Stimulus-response compatibility: An integrated perspective (pp. 31-86). Amsterdam: North-Holland.

Simon, J. R., Acosta, E., JR., Mewaldt, S. P., \& Speidel, C. R. (1976). The effect of an irrelevant directional cue on choice reaction time: Duration of the phenomenon and its relation to stages of processing. Perception \& Psychophysics, 19, 16-22.

Simon, J. R., \& Berbaum, K. (1990). Effect of conflicting cues: The "Stroop effect" vs. the "Simon effect." Acta Psychologica, 73, 159170.

Simon, J. R., \& CRAFT, J. L. (1972). Reaction time in an oddity task: Responding to the different element of a three-light display. Journal of Experimental Psychology, 92, 405-411.

Simon, J. R., Craft, J. L., \& Webster, J. B. (1973). Reactions toward the stimulus source: Analysis of correct responses and errors over a five-day period. Journal of Experimental Psychology, 101, 175-178.

Simon, J. R., Hinrichs, J. V., \& CRAFT, J. L. (1970). Auditory S-R compatibility: Reaction time as a function of ear-hand correspondence and ear-response-location correspondence. Journal of Experimental Psychology, 86, 97-102.

SIMON, J. R., \& RudELl, A. P. (1967). Auditory S-R compatibility: The effect of an irrelevant cue on information processing. Journal of Applied Psychology, 51, 300-304.

Simon, J. R., SLY, P. E., \& VilaPaKKaM, S. (1981). Effect of compatibility of S-R mapping on reaction toward the stimulus source. Acta Psychologica, 47, 63-81.

Simon, J. R., \& Small, A. M., JR. (1969). Processing auditory information: Interference from an irrelevant cue. Journal of Applied Psychology, 53, 433-435.

Simon, J. R., Small, A. M., JR., Ziglar, R. A., \& Craft, J. L. (1970). Response interference in an information processing task: Sensory versus perceptual factors. Journal of Experimental Psychology, 85, 311-314.

SMITH, M. C. (1967). Theories of the psychological refractory period. Psychological Bulletin, 67, 202-213.

SPIJKERS, W. A. C. (1990). Response selection and motor programming: Effects of compatibility and average velocity. In $R$. W. Proctor \& T. G. Reeve (Eds.), Stimulus-response compatibility: An integrated perspective (pp. 297-309). Amsterdam: North-Holland.

STERNBERG, S, (1969). The discovery of processing stages: Extensions of Donders' method. Acta Psychologica, 30, 276-315.

STOFFELS, E. J. (1995). On stage robustness and response selection routes: Further evidence. Manuscript submitted for publication.

Stoffels, E. J., van DeR Molen, M. W., \& Keuss, P. J. G. (1989). An additive factors analysis of the effects of location cues associated with auditory stimuli on stages of information processing. Acta Psychologica, 70, 161-197.

STOFFER, T. (1991). Attentional focusing and spatial stimulus-response compatibility. Psychological Research/Psychologische Forschung, 53, 127-135. 
Stoffer, T., \& YAKIN, A. R. (1994). The functional role of attention for spatial coding in the Simon effect. Psychological Research/Psychologische Forschung, 56, 151-162.

STROOP, J. R. (1992). Studies of interference in serial verbal reactions. Journal of Experimental Psychology: General, 121, 15-23. (Original work published 1935)

SugG, M. J., \& McDonalD, J. E. (1994). Time course of inhibition in color-response and word-response versions of the Stroop task. Journal of Experimental Psychology: Human Perception \& Performance, 20, 647-675.

Treisman, A. M., \& Gelade, G. (1980). A feature-integration theory of attention. Cognitive Psychology, 12, 97-136.

TsaL, Y., \& LAVIE, N. (1993). Location dominance in attending to color and shape. Journal of Experimental Psychology: Human Perception \& Performance, 19, 131-139.

Umiltà, C. (1994). The Simon effect: Introductory remarks. Psychological Research/Psychologische Forschung, 56, 127-129.

UmiLtÀ, C., \& LiotTI, M. (1987). Egocentric and relative spatial codes in S-R compatibility. Psychological Research, 49, 81-90.

Umiltà, C., \& Nicoletti, R. (1985). Attention and coding effects in S-R compatibility due to irrelevant spatial cues. In M. I. Posner \& O. S. M. Marin (Eds.), Attention and performance XI (pp. 456-471). Hillsdale, NJ: Erlbaum.

Umiltà, C., \& Nicoletti, R. (1990). Spatial stimulus-response compatibility. In R. W. Proctor \& T. G. Reeve (Eds.), Stimulus-response compatibility: An integrated perspective (pp. 89-116). Amsterdam North-Holland.

UMiltà, C., \& Nicoletti, R. (1992). An integrated model of the Simon effect. In J. Alegria, D. Holender, J. Junca de Morais, \& M. Radeau (Eds.), Analytic approach to human cognition (pp. 331-350). Amsterdam: Elsevier.

VAN DUREN, L., \& SANDERS, A. F. (1988). On the robustness of the additive factors stage structure in blocked and mixed choice reaction designs. Acta Psychologica, 69, 83-94.

VAN ZANDT, T., \& TowNSEND, J. T. (1993). Self-terminating versus exhaustive processes in rapid visual and memory search: An evaluative review. Perception \& Psychophysics, 53, 563-580.
Verfaellie, M., Bowers, D., \& Heilman, K. M. (1988). Attentional factors in the occurrence of stimulus-response compatibility effects. Neuropsychologia, 26, 435-444.

VIRZI, R. A., \& EGETH, H. E. (1985). Toward a translational model of Stroop interference. Memory \& Cognition, 13, 304-319.

WALLACE, R. J. (1971). S-R compatibility and the idea of a response code. Journal of Experimental Psychology, 88, 354-360.

WALLACE, R. J. (1972). Spatial S-R compatibility effects involving kinesthetic cues. Journal of Experimental Psychology, 93, 163-168.

WARREN, R. E. (1972). Stimulus encoding and memory. Journal of Experimental Psychology, 94, 90-100.

WeEks, D. J., ChUA, R., \& Hambl.IN, K. (in press). Attention shifts and the Simon effect: A failure to replicate Stoffer (1991). Psychological Research/Psychologische Forschung.

WeEKs, D. J., \& Proctor, R. W. (1990). Salient-features coding in the translation between orthogonal stimulus and response dimensions. Journal of Experimental Psychology: General, 119, 355-366.

WhITE, B. W. (1969). Interference in identifying attributes and attribute names. Perception \& Psychophysics, 6, 166-168.

YANTIS, S., \& JoNIDES, J. (1990). Abrupt visual onsets and selective attention: Voluntary versus automatic allocation. Journal of Experimental Psychology: Human Perception \& Performance, 16, 121-134.

\section{NOTES}

1. An earlier study by Simon and Rudell (1967) is sometimes given precedence, but it would be classified as a spatial Stroop task in Kornblum's $(1992,1994)$ taxonomy and according to the terminology used here, because the stimuli in it were the words LEFT and RIGHT.

2 . Location of the precue with respect to fixation was confounded with precue type (stimulus or response location) in Verfaellie et al.'s (1988) experiment and in Proctor et al.'s (1992) direct replication of it. This confound was eliminated in the experiments of Proctor et al. that used only one or the other type of precue.

(Manuscript received June 22, 1994; revision accepted for publication January 18,1995 .) 